\title{
Domain wall partition function of the eight-vertex model with a non-diagonal reflecting end
}

\author{
Wen-Li Yang ${ }^{a}$, Xi Chen ${ }^{a}$, Jun Feng ${ }^{a}$, Kun Hao ${ }^{a}$, Kang-Jie Shi ${ }^{a}$, \\ Cheng-Yi Sun ${ }^{a}$, Zhan-Ying Yang ${ }^{b}$ and Yao-Zhong Zhang ${ }^{c}$ \\ ${ }^{a}$ Institute of Modern Physics, Northwest University, Xian 710069, P.R. China \\ ${ }^{b}$ The Department of Physics, Northwest University, Xian 710069, P.R. China \\ ${ }^{c}$ The University of Queensland, School of Mathematics and Physics, Brisbane, QLD 4072, \\ Australia
}

\begin{abstract}
With the help of the Drinfeld twist or factorizing F-matrix for the eight-vertex SOS model, we obtain the explicit determinant expression of the partition function of the eight-vertex model with a generic non-diagonal reflecting end and domain wall boundary condition. Our result shows that, contrary to the eight-vertex model without a reflection end, the partition function can be expressed as a single determinant.
\end{abstract}

PACS: 03.65.Fd; 04.20.Jb; 05.30.-d; 75.10.Jm

Keywords: The eight-vertex model; Open spin chain; Partition function. 


\section{Introduction}

The domain wall (DW) boundary condition of a statistical model on a finite two-dimensional lattice was first introduced in [1] for the six-vertex model. The partition function of the model (or DW partition function) was then expressed in terms of a determinant [2, 3]. Such a determinant representation of the partition function has played an important role in constructing norms of Bethe states, correction functions [4, 5, 6] and thermodynamical properties of the six-vertex model [7], and also in analyzing the Toda theories [8]. Moreover, it has been proven to be very useful in solving some pure mathematical problems, such as the problem of alternating sign matrices [9]. Recently, the determinant representations of the DW partition function have been obtained for various models [10, 11, 12, 13, 14, 15, 16.

For a two-dimensional statistical model with a reflection end [17], in addition to the local interaction vertex, a reflecting matrix or K-matrix [18] which describes the boundary interactions needs to be introduced at the reflection end of the lattice (see figure 4 below). The DW partition function of the six-vertex model with a diagonal reflection end was exactly calculated and expressed in terms some determinant [17]. Such determinant representation of the model was re-derived [19, 20] by using the Drinfeld twist or factorizing F-matrix [21] method [22]. However, it is highly non-trivial to generalize this result to models with nondiagonal boundary terms [23, 24, 25, 26, 27, 28, 29, 30, 31, 32, 33, 34, 35, 36, 37, 38, 39, 40]. Very recently, determinant representations of DW partition functions have been obtained for the trigonometric SOS model with reflection end [41] and the six-vertex model with a non-diagonal reflection end [42].

Among solvable models, elliptic ones stand out as a particularly important class due to the fact that most trigonometric and rational models can be obtained from them by certain limits. In this paper, we focus on the most fundamental elliptic model - the eight-vertex model [43] whose trigonometric limit gives the six-vertex model. Here we obtain the determinant representation of the DW partition function of the eight-vertex model with a non-diagonal reflection end specified by the generic non-diagonal K-matrix [44, 45]. Our result shows that contrary to the eight-vertex model without reflection end whose DW partition function can only be expressed as a sum of determinants [13, 14, 15], the DW partition function of the eight-vertex model with a non-diagonal reflection end can be represented as a single determinant. Such a single determinant expression will be essential for the study of the 
Bethe vectors of the open XYZ chain with non-diagonal boundary terms.

The paper is organized as follows. In section 2, after introducing our notation and some basic ingredients, we construct the four boundary states which specify the DW boundary condition of the eight-vertex model with a non-diagonal reflection end. In section 3, using the vertex-face correspondence relation we express the DW boundary partition function in terms of the matrix element of the product of the (face type) pseudo-particle creation operators. In section 4, with help of the F-matrix of the eight-vertex SOS model we give the completely symmetric and polarization free representations of the pseudo-particle creation operators in the F-basis. In section 5, we obtain the determinant representation of the DW partition function. In section 6, we summarize our results and give some discussions.

\section{Eight-vertex model with a reflecting end}

In this section, we briefly review the DW boundary condition for the eight-vertex model with non-diagonal reflecting end on an $N \times 2 N$ rectangular lattice.

\subsection{The eight-vertex R-matrix and associated K-matrix}

Let us fix $\tau$ such that $\operatorname{Im}(\tau)>0$ and $\Lambda_{\tau}$ be the lattice generated by 1 and $\tau$. Introduce the following elliptic functions

$$
\begin{aligned}
\theta\left[\begin{array}{l}
a \\
b
\end{array}\right](u, \tau) & =\sum_{n=-\infty}^{\infty} \exp \left\{i \pi\left[(n+a)^{2} \tau+2(n+a)(u+b)\right]\right\}, \\
\theta^{(j)}(u) & =\theta\left[\begin{array}{c}
\frac{1}{2}-\frac{j}{2} \\
\frac{1}{2}
\end{array}\right](u, 2 \tau), \quad j=1,2 ; \quad \sigma(u)=\theta\left[\begin{array}{c}
\frac{1}{2} \\
\frac{1}{2}
\end{array}\right](u, \tau) .
\end{aligned}
$$

The $\sigma$-function 1 satisfies the so-called Riemann identity:

$$
\begin{aligned}
\sigma(u+ & x) \sigma(u-x) \sigma(v+y) \sigma(v-y)-\sigma(u+y) \sigma(u-y) \sigma(v+x) \sigma(v-x) \\
& =\sigma(u+v) \sigma(u-v) \sigma(x+y) \sigma(x-y)
\end{aligned}
$$

which will be useful in the following. Moreover, for any $\alpha=\left(\alpha_{1}, \alpha_{2}\right), \alpha_{1}, \alpha_{2} \in \mathbb{Z}_{2}$, we can introduce a function $\sigma_{\alpha}(u)$ as follow

$$
\sigma_{\alpha}(u)=\theta\left[\begin{array}{c}
\frac{1}{2}+\frac{\alpha_{1}}{2} \\
\frac{1}{2}+\frac{\alpha_{2}}{2}
\end{array}\right](u, \tau), \quad \alpha_{1}, \alpha_{2} \in \mathbb{Z}_{2} .
$$

\footnotetext{
${ }^{1}$ Our $\sigma$-function is the $\vartheta$-function $\vartheta_{1}(u)$ [46. It has the following relation with the Weierstrassian $\sigma$ function $\sigma_{w}(u): \sigma_{w}(u) \propto e^{\eta_{1} u^{2}} \sigma(u)$ with $\eta_{1}=\pi^{2}\left(\frac{1}{6}-4 \sum_{n=1}^{\infty} \frac{n q^{2 n}}{1-q^{2 n}}\right)$ and $q=e^{i \tau}$.
} 
The above definition implies the identification $\sigma_{(0,0)}(u)=\sigma(u)$.

Let $V$ be a two-dimensional vector space $\mathbb{C}^{2}$ and $\left\{\epsilon_{i} \mid i=1,2\right\}$ be the orthonormal basis of $V$ such that $\left\langle\epsilon_{i}, \epsilon_{j}\right\rangle=\delta_{i j}$. The well-known eight-vertex model R-matrix $\bar{R}(u) \in \operatorname{End}(V \otimes V)$ is given by

$$
\bar{R}(u)=\left(\begin{array}{llll}
a(u) & & & d(u) \\
& b(u) & c(u) & \\
& c(u) & b(u) & \\
d(u) & & & a(u)
\end{array}\right)
$$

The non-vanishing matrix elements are [43]

$$
\begin{array}{ll}
a(u)=\frac{\theta^{(1)}(u) \theta^{(0)}(u+\eta) \sigma(\eta)}{\theta^{(1)}(0) \theta^{(0)}(\eta) \sigma(u+\eta)}, & b(u)=\frac{\theta^{(0)}(u) \theta^{(1)}(u+\eta) \sigma(\eta)}{\theta^{(1)}(0) \theta^{(0)}(\eta) \sigma(u+\eta)}, \\
c(u)=\frac{\theta^{(1)}(u) \theta^{(1)}(u+\eta) \sigma(\eta)}{\theta^{(1)}(0) \theta^{(1)}(\eta) \sigma(u+\eta)}, & d(u)=\frac{\theta^{(0)}(u) \theta^{(0)}(u+\eta) \sigma(\eta)}{\theta^{(1)}(0) \theta^{(1)}(\eta) \sigma(u+\eta)} .
\end{array}
$$

Here $u$ is the spectral parameter and $\eta$ is the so-called crossing parameter. The R-matrix satisfies the quantum Yang-Baxter equation (QYBE)

$$
\bar{R}_{1,2}\left(u_{1}-u_{2}\right) \bar{R}_{1,3}\left(u_{1}-u_{3}\right) \bar{R}_{2,3}\left(u_{2}-u_{3}\right)=\bar{R}_{2,3}\left(u_{2}-u_{3}\right) \bar{R}_{1,3}\left(u_{1}-u_{3}\right) \bar{R}_{1,2}\left(u_{1}-u_{2}\right) .
$$

Throughout we adopt the standard notation: for any matrix $A \in \operatorname{End}(V), A_{j}$ (or $\left.A^{j}\right)$ is an embedding operator in the tensor space $V \otimes V \otimes \cdots$, which acts as $A$ on the $j$-th space and as identity on the other factor spaces; $R_{i, j}(u)$ is an embedding operator of R-matrix in the tensor space, which acts as identity on the factor spaces except for the $i$-th and $j$-th ones.

For a model with reflection end [18, in addition to the R-matrix, one needs to introduce K-matrix $K(u)$ which satisfies the reflection equation [47] (RE)

$$
\begin{aligned}
& \bar{R}_{1,2}\left(u_{1}-u_{2}\right) K_{1}\left(u_{1}\right) \bar{R}_{2,1}\left(u_{1}+u_{2}\right) K_{2}\left(u_{2}\right) \\
& \quad=K_{2}\left(u_{2}\right) \bar{R}_{1,2}\left(u_{1}+u_{2}\right) K_{1}\left(u_{1}\right) \bar{R}_{2,1}\left(u_{1}-u_{2}\right) .
\end{aligned}
$$

In this paper, we consider the K-matrix $K(u)$ which is a generic solution [44, 45] to the RE (2.8) associated with the R-matrix (2.5),

$$
K(u)=k_{0}(u)+k_{x}(u) \sigma^{x}+k_{y} \sigma^{y}+k_{z}(u) \sigma^{z},
$$

where $\sigma^{x}, \sigma^{y}, \sigma^{z}$ are the Pauli matrices and the coefficient functions are

$$
k_{0}(u)=\frac{\sigma(2 u) \sigma\left(\lambda_{1}+\lambda_{2}-\frac{1}{2}\right) \sigma\left(\lambda_{1}+\zeta\right) \sigma\left(\lambda_{2}+\zeta\right)}{2 \sigma(u) \sigma\left(-u+\lambda_{1}+\lambda_{2}-\frac{1}{2}\right) \sigma\left(\lambda_{1}+\zeta+u\right) \sigma\left(\lambda_{2}+\zeta+u\right)},
$$




$$
\begin{aligned}
& k_{x}(u)=\frac{\sigma(2 u) \sigma_{(1,0)}\left(\lambda_{1}+\lambda_{2}-\frac{1}{2}\right) \sigma_{(1,0)}\left(\lambda_{1}+\zeta\right) \sigma_{(1,0)}\left(\lambda_{2}+\zeta\right)}{2 \sigma_{(1,0)}(u) \sigma\left(-u+\lambda_{1}+\lambda_{2}-\frac{1}{2}\right) \sigma\left(\lambda_{1}+\zeta+u\right) \sigma\left(\lambda_{2}+\zeta+u\right)}, \\
& k_{y}(u)=\frac{i \sigma(2 u) \sigma_{(1,1)}\left(\lambda_{1}+\lambda_{2}-\frac{1}{2}\right) \sigma_{(1,1)}\left(\lambda_{1}+\zeta\right) \sigma_{(1,1)}\left(\lambda_{2}+\zeta\right)}{2 \sigma_{(1,1)}(u) \sigma\left(-u+\lambda_{1}+\lambda_{2}-\frac{1}{2}\right) \sigma\left(\lambda_{1}+\zeta+u\right) \sigma\left(\lambda_{2}+\zeta+u\right)}, \\
& k_{z}(u)=\frac{\sigma(2 u) \sigma_{(0,1)}\left(\lambda_{1}+\lambda_{2}-\frac{1}{2}\right) \sigma_{(0,1)}\left(\lambda_{1}+\zeta\right) \sigma_{(0,1)}\left(\lambda_{2}+\zeta\right)}{2 \sigma_{(0,1)}(u) \sigma\left(-u+\lambda_{1}+\lambda_{2}-\frac{1}{2}\right) \sigma\left(\lambda_{1}+\zeta+u\right) \sigma\left(\lambda_{2}+\zeta+u\right)} .
\end{aligned}
$$

Here there are 3 free boundary parameters $\left\{\lambda_{1}, \lambda_{2}, \zeta\right\}$, which are related to different boundary interactions. It is very convenient to introduce a vector $\lambda \in V$ associated with the boundary parameters $\left\{\lambda_{i}\right\}$,

$$
\lambda=\sum_{k=1}^{2} \lambda_{k} \epsilon_{k}
$$

\subsection{The model}

The partition function of a statistical model on a two-dimensional lattice is defined by

$$
Z=\sum \exp \left\{-\frac{E}{k T}\right\}
$$

where $E$ is the energy of the system, $k$ is the Boltzmann constant, $T$ is the temperature of the system, and the summation is taken over all possible configurations under certain boundary condition such as the DW boundary condition. The model we consider here has eight allowed bulk vertex configurations
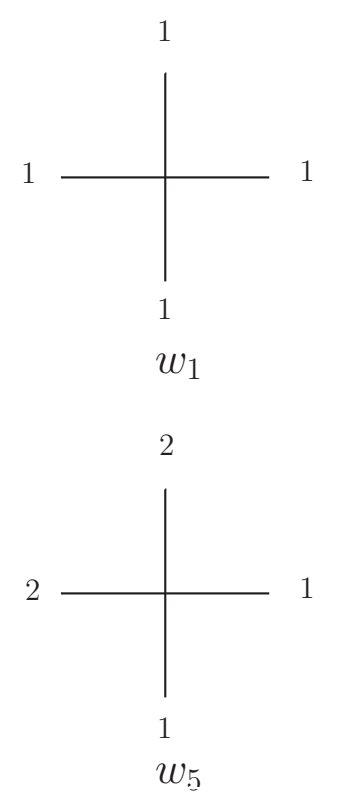
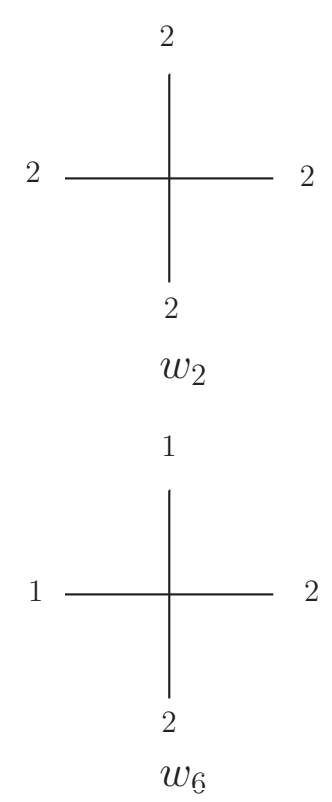

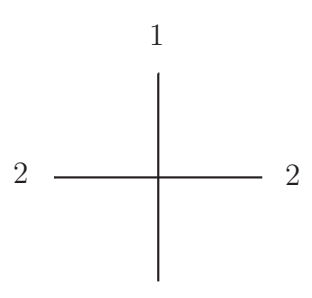

1

$w_{3}$

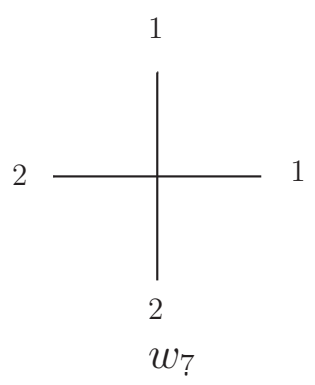

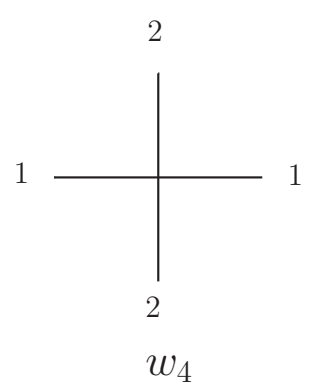

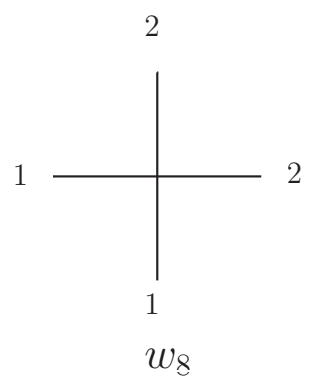


Figure 1. Vertex configurations and their associated Boltzmann weights.

and four allowed configurations at each reflection end
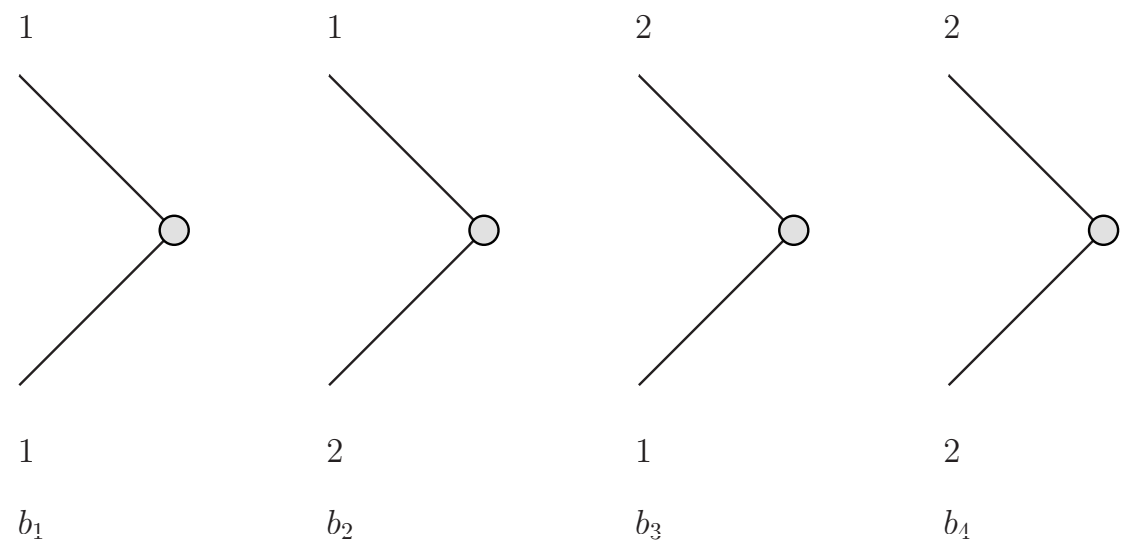

Figure 2. Reflection ends and the associated Boltzmann weights.

where 1 and 2 respectively denote the spin up and down states. Each of the eight bulk configurations is assigned a statistical weight (or Boltzmann weight) $w_{i}$, while each of the four reflection configurations is assigned a weight $b_{i}$. Then the partition function of the model with a reflection end can be rewritten as

$$
Z=\sum w_{1}^{n_{1}} w_{2}^{n_{2}} w_{3}^{n_{3}} w_{4}^{n_{4}} w_{5}^{n_{5}} w_{6}^{n_{6}} w_{7}^{n_{7}} w_{8}^{n_{8}} b_{1}^{l_{1}} b_{2}^{l_{2}} b_{3}^{l_{3}} b_{4}^{l_{4}}
$$

where the summation is over all possible configurations with $n_{i}$ and $l_{j}$ being the number of vertices of type $i$ and the number of reflection ends of type $j$ respectively. The bulk Boltzmann weights which we consider here have $Z_{2}$-symmetry, i.e.,

$$
a \equiv w_{1}=w_{2}, \quad b \equiv w_{3}=w_{4}, \quad c \equiv w_{5}=w_{6}, \quad d \equiv w_{7}=w_{8},
$$

and variables $a, b, c, d$ satisfy a function relation, or equivalently, the local Boltzmann weights $\left\{w_{i}\right\}$ can be parameterized by the matrix elements of the eight-vertex R-matrix (2.5) as in figure 3. At the same time, the weights $\left\{b_{i}\right\}$ corresponding to the reflection end can be parameterized by the matrix elements of the corresponding K-matrix (2.9) as in figure 3. 


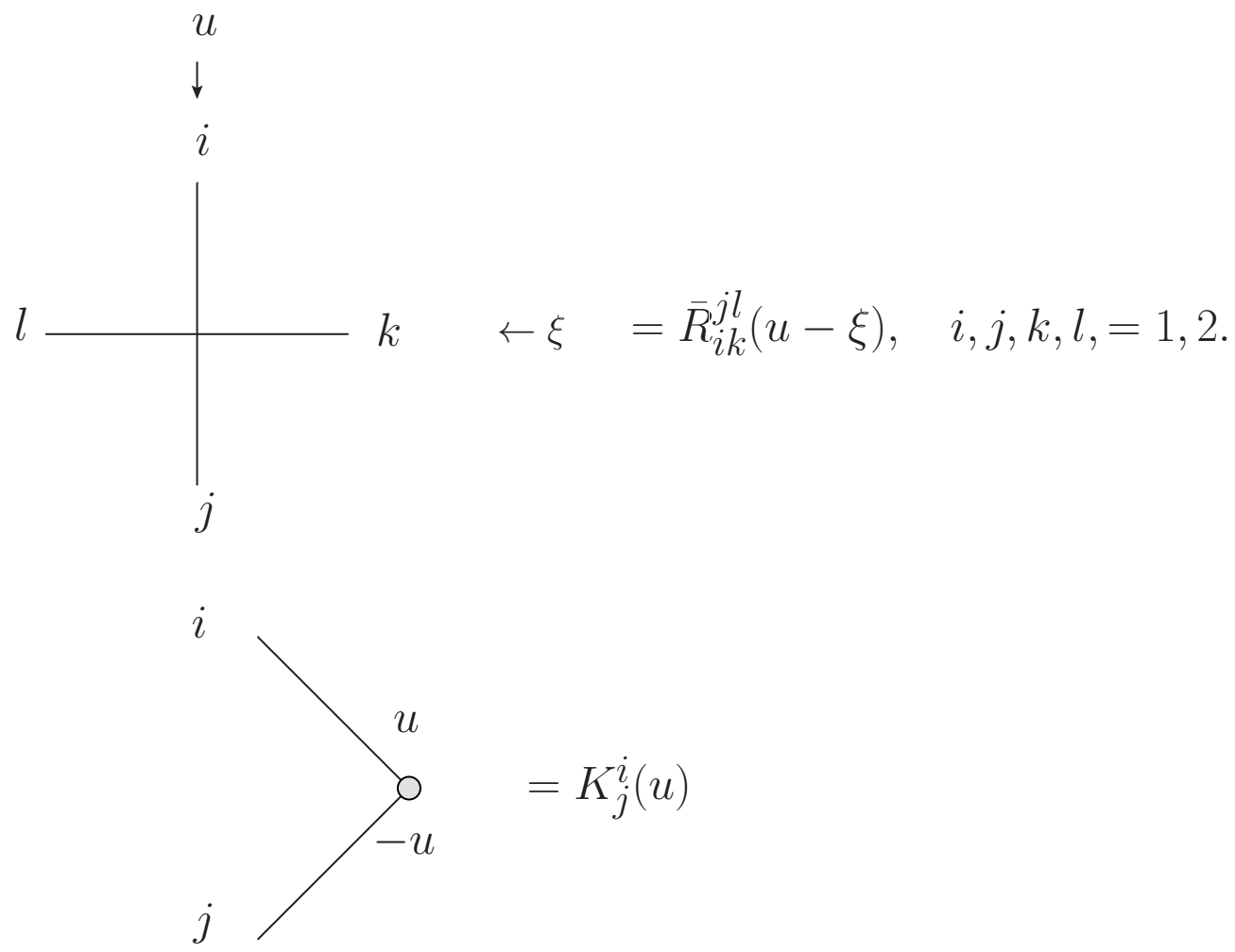

Figure 3. The Boltzmann weights and elements of the eight-vertex R-matrix and K-matrix.

Then the corresponding model is called the eight-vertex model with a reflection end. Its partition function is thus given by

$$
Z=\sum a^{n_{1}+n_{2}} b^{n_{3}+n_{4}} c^{n_{5}+n_{6}} d^{n_{7}+n_{8}} b_{1}^{l_{1}} b_{2}^{l_{2}} b_{3}^{l_{3}} b_{4}^{l_{4}}
$$

As shown in figure 3, to parameterize the bulk Boltzmann weights in terms of the elements of the R-matrix, one needs to assign spectral parameters $u$ and $\xi$ respectively to the vertical and horizontal lines of each vertex of the lattice. In an inhomogeneous model, the statistical weights are site-dependent. Hence two sets of spectral parameters $\left\{u_{\alpha}\right\}$ and $\left\{\xi_{i}\right\}$ are needed, see figure 4 . The horizontal lines are enumerated by indices $1, \ldots, N$ with spectral parameters $\left\{\xi_{i}\right\}$, while the vertical lines are enumerated by indices $\overline{1}, \ldots, \bar{N}$ with spectral parameters $\left\{\bar{u}_{\alpha}\right\}$ (The $2 N$ parameters $\left\{\bar{u}_{\alpha}\right\}$ are assigned as follow: $\bar{u}_{2 i}=u_{i}$ and $\bar{u}_{2 i+1}=-u_{i}$, as shown in figure 4.). The DW boundary condition is specified by four boundary states $\left|\Omega^{(2)}(\lambda)\right\rangle$, $\left|\bar{\Omega}^{(1)}(\lambda)\right\rangle,\left\langle\Omega^{(1)}(\lambda)\right|$ and $\left\langle\bar{\Omega}^{(2)}(\lambda)\right|$ (the definitions of the boundary states will be given later, see (2.38)-(2.41) below). These four states correspond to the particular choices of spin states on the four boundaries of the lattice . 


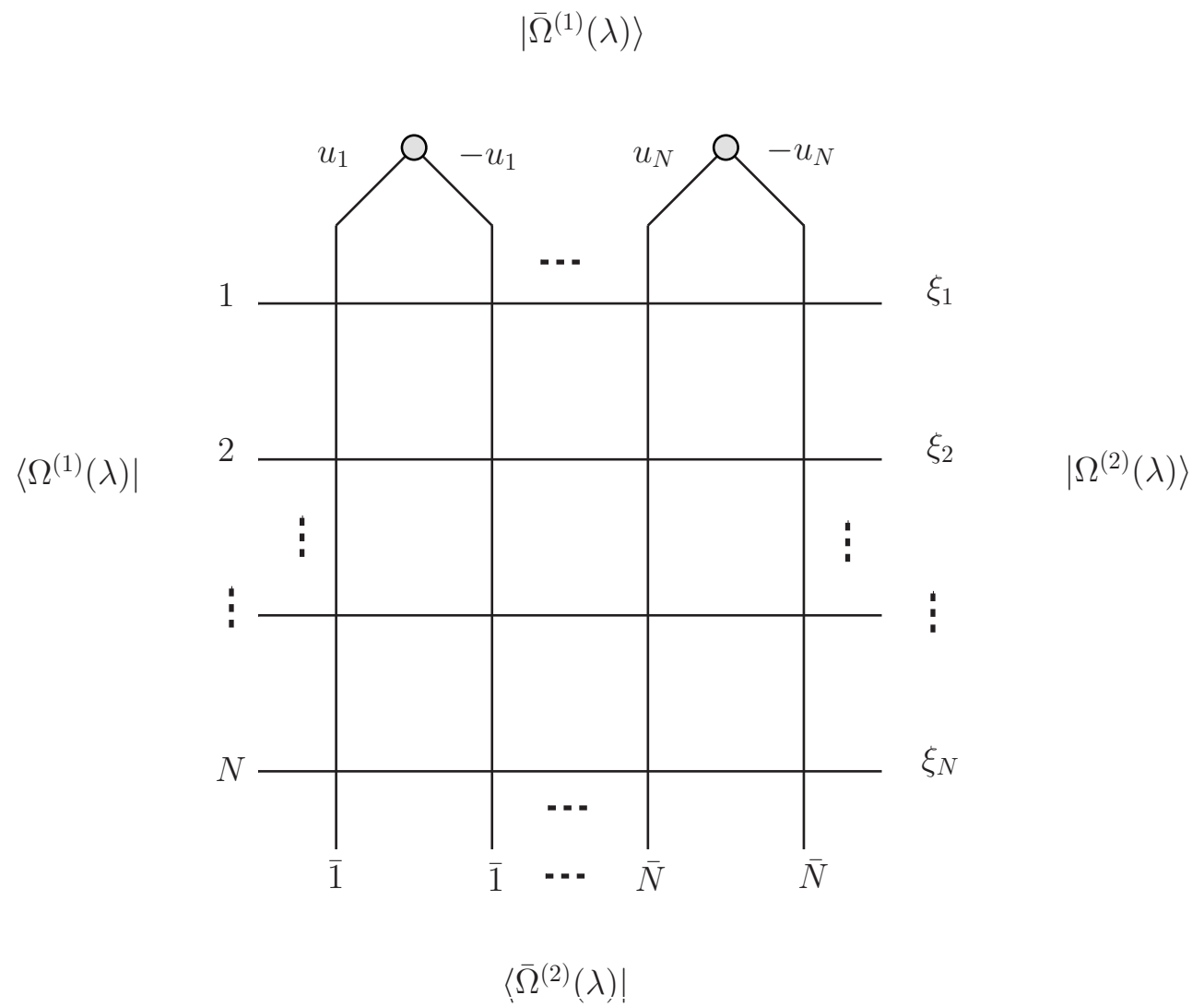

Figure 4. The eight-vertex model with a non-diagonal reflection end and the DW condition.

Some remarks are in order. The boundary states not only depend on the spectral parameters $\left(\left|\Omega^{(2)}(\lambda)\right\rangle\right.$ and $\left\langle\Omega^{(1)}(\lambda)\right|$ depend on $\left\{\xi_{i}\right\}$, while $\left|\bar{\Omega}^{(1)}(\lambda)\right\rangle$ and $\left\langle\bar{\Omega}^{(2)}(\lambda)\right|$ depend on $\left.\left\{u_{\alpha}\right\}\right)$ but also on two continuous parameters $\lambda_{1}$ and $\lambda_{2}$. However, in the trigonometric limit (i.e., setting $\lambda_{2}=\frac{\tau}{2}$ and then taking $\left.\tau \rightarrow+i \infty\right)$, the corresponding boundary states $\left|\Omega^{(1)}(\lambda)\right\rangle$ and $\left\langle\bar{\Omega}^{(1)}(\lambda)\right|$ (or $\left|\bar{\Omega}^{(2)}(\lambda)\right\rangle$ and $\left.\left\langle\Omega^{(2)}(\lambda)\right|\right)$ become the state of all spin up and its dual (or the state of all spin down and its dual) up to some over-all scalar factors.

The partition function of the eight-vertex model with a non-diagonal reflection end specified by the generic K-matrix $K(u)(2.9)$ and the DW boundary condition is a function of $2 N+3$ variables $\left\{u_{\alpha}\right\},\left\{\xi_{i}\right\}, \lambda_{1}, \lambda_{2}$ and $\zeta$, which is denoted by $Z_{N}\left(\left\{u_{\alpha}\right\} ;\left\{\xi_{i}\right\} ; \lambda ; \zeta\right)$. Due to the fact that the local Boltzmann weights of each vertex and reflection end of the lattice are given by the matrix elements of the eight-vertex R-matrix and the associated K-matrix (see figure 3), the partition function can be expressed in terms of the product of the R-matrices, the K-matrices and the four boundary states

$Z_{N}\left(\left\{u_{\alpha}\right\} ;\left\{\xi_{i}\right\} ; \lambda ; \zeta\right)=\left\langle\Omega^{(1)}(\lambda)\right|\left\langle\bar{\Omega}^{(2)}(\lambda)\right|$ 


$$
\begin{aligned}
& \times \bar{R}_{\overline{1}, N}\left(u_{1}-\xi_{N}\right) \ldots \bar{R}_{\overline{1}, 1}\left(u_{1}-\xi_{1}\right) K_{\overline{1}}\left(u_{1}\right) \bar{R}_{1, \overline{1}}\left(u_{1}+\xi_{1}\right) \ldots \bar{R}_{N, \overline{1}}\left(u_{1}+\xi_{N}\right) \\
& \quad \vdots \\
& \times \bar{R}_{\bar{N}, N}\left(u_{N}-\xi_{N}\right) \ldots \bar{R}_{\bar{N}, 1}\left(u_{N}-\xi_{1}\right) K_{\bar{N}}\left(u_{N}\right) \bar{R}_{1, \bar{N}}\left(u_{N}+\xi_{1}\right) \ldots \bar{R}_{N, \bar{N}}\left(u_{N}+\xi_{N}\right) \\
& \times\left|\bar{\Omega}^{(1)}(\lambda)\right\rangle\left|\Omega^{(2)}(\lambda)\right\rangle .
\end{aligned}
$$

One can rearrange the product of the R-matrices in (2.13) in terms of a product of the so-called double-row monodromy matrices

$$
Z_{N}\left(\left\{u_{\alpha}\right\} ;\left\{\xi_{i}\right\} ; \lambda ; \zeta\right)=\left\langle\Omega^{(1)}(\lambda)\left|\left\langle\bar{\Omega}^{(2)}(\lambda)\left|\mathbb{T}_{\overline{1}}\left(u_{1}\right) \ldots \mathbb{T}_{\bar{N}}\left(u_{N}\right)\right| \bar{\Omega}^{(1)}(\lambda)\right\rangle\right| \Omega^{(2)}(\lambda)\right\rangle
$$

where the monodromy matrix $\mathbb{T}_{\bar{i}}(u)$ is given by

$$
\begin{aligned}
\mathbb{T}_{\bar{i}}(u) \equiv \mathbb{T}_{\bar{i}}\left(u ; \xi_{1}, \ldots, \xi_{N} ; \zeta\right)= & \bar{R}_{\bar{i}, N}\left(u_{i}-\xi_{N}\right) \ldots \bar{R}_{\bar{i}, 1}\left(u_{i}-\xi_{1}\right) \\
& \times K_{\bar{i}}\left(u_{i}\right) \bar{R}_{1, \bar{i}}\left(u_{i}+\xi_{1}\right) \ldots \bar{R}_{N, \bar{i}}\left(u_{i}+\xi_{N}\right) .
\end{aligned}
$$

The double-row matrix $\mathbb{T}(u)$ has played an important role in constructing the transfer matrix for an open spin chain [18]. The QYBE (2.7) of the R-matrix and the reflection equation (2.8) of the K-matrix ensure that the monodromy matrix $\mathbb{T}_{\bar{i}}(u)$ satisfy the following exchange relation

$$
\bar{R}_{\bar{i}, \bar{j}}\left(u_{i}-u_{j}\right) \mathbb{T}_{\bar{i}}\left(u_{i}\right) \bar{R}_{\bar{j}, \bar{i}}\left(u_{i}+u_{j}\right) \mathbb{T}_{\bar{j}}\left(u_{j}\right)=\mathbb{T}_{\bar{j}}\left(u_{j}\right) \bar{R}_{\bar{i}, \bar{j}}\left(u_{i}+u_{j}\right) \mathbb{T}_{\bar{i}}\left(u_{i}\right) \bar{R}_{\bar{j}, \bar{i}}\left(u_{i}-u_{j}\right) .
$$

\subsection{The boundary states}

From the orthonormal basis $\left\{\epsilon_{i}\right\}$ of $V$, we define

$$
\hat{\imath}=\epsilon_{i}-\bar{\epsilon}, \quad \bar{\epsilon}=\frac{1}{2} \sum_{k=1}^{2} \epsilon_{k}, \quad i=1,2, \quad \text { then } \sum_{i=1}^{2} \hat{\imath}=0 .
$$

Let $\mathfrak{h}$ be the Cartan subalgebra of $A_{1}$ and $\mathfrak{h}^{*}$ be its dual. A finite-dimensional diagonalizable $\mathfrak{h}$-module is a complex finite-dimensional vector space $W$ with a weight decomposition $W=$ $\oplus_{\mu \in \mathfrak{h}^{*}} W[\mu]$, so that $\mathfrak{h}$ acts on $W[\mu]$ by $x v=\mu(x) v,(x \in \mathfrak{h}, v \in W[\mu])$. For example, the non-zero weight spaces of the fundamental representation $V_{\Lambda_{1}}=\mathbb{C}^{2}=V$ are

$$
W[\hat{\imath}]=\mathbb{C}_{i}, \quad i=1,2 .
$$

For a generic $m \in V$, define

$$
m_{i}=\left\langle m, \epsilon_{i}\right\rangle, \quad m_{i j}=m_{i}-m_{j}=\left\langle m, \epsilon_{i}-\epsilon_{j}\right\rangle, \quad i, j=1,2 .
$$


Let $R(u, m) \in \operatorname{End}(V \otimes V)$ be the R-matrix of the eight-vertex SOS model [43] given by

$$
R(u ; m)=\sum_{i=1}^{2} R(u ; m)_{i i}^{i i} E_{i i} \otimes E_{i i}+\sum_{i \neq j}^{2}\left\{R(u ; m)_{i j}^{i j} E_{i i} \otimes E_{j j}+R(u ; m)_{i j}^{j i} E_{j i} \otimes E_{i j}\right\},
$$

where $E_{i j}$ is the matrix with elements $\left(E_{i j}\right)_{k}^{l}=\delta_{j k} \delta_{i l}$. The coefficient functions are

$$
\begin{aligned}
& R(u ; m)_{i i}^{i i}=1, \quad R(u ; m)_{i j}^{i j}=\frac{\sigma(u) \sigma\left(m_{i j}-\eta\right)}{\sigma(u+\eta) \sigma\left(m_{i j}\right)}, \quad i \neq j, \\
& R(u ; m)_{i j}^{j i}=\frac{\sigma(\eta) \sigma\left(u+m_{i j}\right)}{\sigma(u+\eta) \sigma\left(m_{i j}\right)}, \quad i \neq j,
\end{aligned}
$$

and $m_{i j}$ is defined in (2.19). The R-matrix satisfies the dynamical (modified) quantum Yang-Baxter equation (or the star-triangle relation) [43]

$$
\begin{array}{r}
R_{1,2}\left(u_{1}-u_{2} ; m-\eta h^{(3)}\right) R_{1,3}\left(u_{1}-u_{3} ; m\right) R_{2,3}\left(u_{2}-u_{3} ; m-\eta h^{(1)}\right) \\
=R_{2,3}\left(u_{2}-u_{3} ; m\right) R_{1,3}\left(u_{1}-u_{3} ; m-\eta h^{(2)}\right) R_{1,2}\left(u_{1}-u_{2} ; m\right) .
\end{array}
$$

Here we have adopted the convention

$$
R_{1,2}\left(u, m-\eta h^{(3)}\right) v_{1} \otimes v_{2} \otimes v_{3}=(R(u, m-\eta \mu) \otimes \text { id }) v_{1} \otimes v_{2} \otimes v_{3}, \quad \text { if } v_{3} \in W[\mu] .
$$

Moreover, one may check that the R-matrix satisfies the weight conservation condition,

$$
\left[h^{(1)}+h^{(2)}, R_{1,2}(u ; m)\right]=0,
$$

the unitary condition,

$$
R_{1,2}(u ; m) R_{2,1}(-u ; m)=\mathrm{id} \otimes \mathrm{id}
$$

and the crossing relation

$$
R(u ; m)_{i j}^{k l}=\varepsilon_{l} \varepsilon_{j} \frac{\sigma(u) \sigma\left((m-\eta \hat{\imath})_{21}\right)}{\sigma(u+\eta) \sigma\left(m_{21}\right)} R(-u-\eta ; m-\eta \hat{\imath})_{\bar{l} i}^{\bar{j} k},
$$

where

$$
\varepsilon_{1}=1, \varepsilon_{2}=-1, \quad \text { and } \overline{1}=2, \overline{2}=1 \text {. }
$$

Let us introduce two intertwiners which are 2-component column vectors $\phi_{m, m-\eta \hat{\jmath}}(u)$ labelled by $\hat{1}, \hat{2}$. The $k$-th element of $\phi_{m, m-\eta \hat{\jmath}}(u)$ is given by

$$
\phi_{m, m-\eta \hat{\jmath}}^{(k)}(u)=\theta^{(k)}\left(u+2 m_{j}\right)
$$


where the functions $\theta^{(j)}(u)$ are given in (2.2). Explicitly,

$$
\phi_{m, m-\eta \hat{1}}(u)=\left(\begin{array}{c}
\theta^{(1)}\left(u+2 m_{1}\right) \\
\theta^{(2)}\left(u+2 m_{1}\right)
\end{array}\right), \quad \phi_{m, m-\eta \hat{2}}(u)=\left(\begin{array}{c}
\theta^{(1)}\left(u+2 m_{2}\right) \\
\theta^{(2)}\left(u+2 m_{2}\right)
\end{array}\right) .
$$

One can prove the following identity [48]

$$
\operatorname{det}\left|\begin{array}{ll}
\theta^{(1)}\left(u+2 m_{1}\right) & \theta^{(1)}\left(u+2 m_{2}\right) \\
\theta^{(2)}\left(u+2 m_{1}\right) & \theta^{(2)}\left(u+2 m_{2}\right)
\end{array}\right|=C(\tau) \sigma\left(u+m_{1}+m_{2}-\frac{1}{2}\right) \sigma\left(m_{12}\right),
$$

where $C(\tau)$ is non-vanishing constant which depends on $\tau$. This implies that the two intertwiner vectors $\phi_{m, m-\eta \hat{\imath}}(u)$ are linearly independent for a generic $m \in V$.

Using the intertwiner vectors, one can derive the following face-vertex correspondence relation 43

$$
\begin{aligned}
\bar{R}_{1,2}\left(u_{1}-u_{2}\right) \phi_{m, m-\eta \hat{\imath}}^{1}\left(u_{1}\right) \phi_{m-\eta \hat{\imath}, m-\eta(\hat{\imath}+\hat{\jmath})}^{2}\left(u_{2}\right) \\
\quad=\sum_{k, l} R\left(u_{1}-u_{2} ; m\right)_{i j}^{k l} \phi_{m-\eta \hat{l}, m-\eta(\hat{l}+\hat{k})}^{1}\left(u_{1}\right) \phi_{m, m-\eta \hat{l}}^{2}\left(u_{2}\right) .
\end{aligned}
$$

Then the QYBE (2.7) of the vertex-type R-matrix $\bar{R}(u)$ is equivalent to the dynamical YangBaxter equation (2.23) of the SOS R-matrix $R(u, m)$. For a generic $m$, we can introduce other types of intertwiners $\bar{\phi}, \tilde{\phi}$ which are both row vectors and satisfy the following conditions,

$$
\bar{\phi}_{m, m-\eta \hat{\mu}}(u) \phi_{m, m-\eta \hat{\nu}}(u)=\delta_{\mu \nu}, \quad \tilde{\phi}_{m+\eta \hat{\mu}, m}(u) \phi_{m+\eta \hat{\nu}, m}(u)=\delta_{\mu \nu}
$$

from which one can derive the relations,

$$
\begin{aligned}
& \sum_{\mu=1}^{2} \phi_{m, m-\eta \hat{\mu}}(u) \bar{\phi}_{m, m-\eta \hat{\mu}}(u)=\mathrm{id}, \\
& \sum_{\mu=1}^{2} \phi_{m+\eta \hat{\mu}, m}(u) \tilde{\phi}_{m+\eta \hat{\mu}, m}(u)=\mathrm{id} .
\end{aligned}
$$

Following the method in [48], we check that the K-matrices $K(u)$ given by (2.9) can be expressed in terms of the intertwiners and diagonal matrices $\mathcal{K}(\lambda \mid u)$ as follows

$$
K(u)_{t}^{s}=\sum_{i, j} \phi_{\lambda-\eta(\hat{\imath}-\hat{\jmath}), \lambda-\eta \hat{\imath}}^{(s)}(u) \mathcal{K}(\lambda \mid u)_{i}^{j} \bar{\phi}_{\lambda, \lambda-\eta \hat{\imath}}^{(t)}(-u) .
$$

Here the diagonal matrix $\mathcal{K}(\lambda \mid u)$ is given by

$$
\mathcal{K}(\lambda \mid u) \equiv \operatorname{Diag}\left(k(\lambda \mid u)_{1}, k(\lambda \mid u)_{2}\right)=\operatorname{Diag}\left(\frac{\sigma\left(\lambda_{1}+\zeta-u\right)}{\sigma\left(\lambda_{1}+\zeta+u\right)}, \frac{\sigma\left(\lambda_{2}+\zeta-u\right)}{\sigma\left(\lambda_{2}+\zeta+u\right)}\right) .
$$


Although the vertex type K-matrix $K^{-}(u)$ given by (2.9) is generally non-diagonal, after the face-vertex transformation (2.36), the face type counterpart $\mathcal{K}(\lambda \mid u)$ becomes diagonal. This fact enabled the authors in [26, 38, to diagonalize the transfer matrix of the open chains with non-diagonal terms by applying the generalized algebraic Bethe ansatz method developed in [28].

Now we are in the position to construct the boundary states specifying the DW boundary condition of the eight-vertex model with a non-diagonal reflection end, see figure 4 . For any vector $m \in V$, we introduce four states which live in the two N-tensor spaces of $V$ (one is indexed by $1, \ldots, N$ and the other is indexed by $\overline{1}, \ldots, \bar{N})$ or their dual spaces as follows:

$$
\begin{aligned}
\left|\Omega^{(2)}(m)\right\rangle= & \phi_{m, m-\eta \hat{2}}^{1}\left(\xi_{1}\right) \phi_{m-\eta \hat{\imath}, m-2 \eta \hat{2}}^{2}\left(\xi_{2}\right) \ldots \phi_{m-\eta(N-1) \hat{2}, m-\eta N \hat{2}}^{N}\left(\xi_{N}\right), \\
\left|\bar{\Omega}^{(1)}(m)\right\rangle= & \phi_{m-(N-2) \eta \hat{1}, m-(N-2) \eta \hat{1}-\eta \hat{1}}^{\overline{1}}\left(-u_{1}\right) \phi_{m-(N-4) \eta \hat{1}, m-(N-4) \eta \hat{1}-\eta \hat{1}}^{\overline{1}}\left(-u_{2}\right) \\
& \times \ldots \phi_{m+N \eta \hat{1}, m+N \eta \hat{1}-\eta \hat{1}}^{\bar{N}}\left(-u_{N}\right), \\
\left\langle\Omega^{(1)}(m)\right|= & \tilde{\phi}_{m, m-\eta \hat{1}}^{1}\left(\xi_{1}\right) \tilde{\phi}_{m-\eta \hat{1}, m-2 \eta \hat{1}}^{2}\left(\xi_{2}\right) \ldots \tilde{\phi}_{m-\eta(N-1) \hat{1}, m-\eta N \hat{1}}^{N}\left(\xi_{N}\right), \\
\left\langle\bar{\Omega}^{(2)}(m)\right|= & \tilde{\phi}_{m-N \eta \hat{1}, m-N \eta \hat{1}-\eta \hat{2}}^{\overline{1}}\left(u_{1}\right) \tilde{\phi}_{m-(N-2) \eta \hat{1}, m-(N-2) \eta \hat{1}-\eta \hat{2}}^{\overline{1}}\left(u_{2}\right) \\
& \times \ldots \tilde{\phi}_{m+\eta(N-2) \hat{1}, m-\eta(N-2) \hat{1}-\eta \hat{2}}^{\bar{N}}\left(u_{N}\right) .
\end{aligned}
$$

The boundary states corresponding to the DW boundary condition can be obtained through the above states by special choices of $m$ and $i$ (for example, $m$ is specified to $\lambda$ which is related to the parameters of the K-matrix $K(u))$. The DW partition function $Z_{N}\left(\left\{u_{\alpha}\right\} ;\left\{\xi_{i}\right\} ; \lambda ; \zeta\right)$ (2.13) of the eight-vertex model with a non-diagonal reflection end becomes

$$
\begin{aligned}
& Z_{N}\left(\left\{u_{\alpha}\right\} ;\left\{\xi_{i}\right\} ; \lambda ; \zeta\right)= \\
& \quad \tilde{\phi}_{\lambda, \lambda-\eta \hat{1}}^{1}\left(\xi_{1}\right) \ldots \tilde{\phi}_{\lambda-(N-1) \eta \hat{1}, \lambda-N \eta \hat{1}}^{N}\left(\xi_{N}\right) \tilde{\phi}_{\lambda-N \eta \hat{1}, \lambda-N \eta \hat{1}-\eta \hat{2}}^{\overline{1}}\left(u_{1}\right) \ldots \tilde{\phi}_{\lambda+(N-2) \eta \hat{1}, \lambda+(N-2) \eta \hat{1}-\eta \hat{2}}^{\bar{N}}\left(u_{N}\right) \\
& \quad \times R_{\overline{1}, N}\left(u_{1}-\xi_{N}\right) \ldots R_{\overline{1}, 1}\left(u_{1}-\xi_{1}\right) K_{\overline{1}}\left(u_{1}\right) R_{1, \overline{1}}\left(u_{1}+\xi_{1}\right) \ldots R_{N, \overline{1}}\left(u_{1}+\xi_{N}\right) \\
& \quad \quad \vdots \\
& \quad \times R_{\bar{N}, N}\left(u_{N}-\xi_{N}\right) \ldots R_{\bar{N}, 1}\left(u_{N}-\xi_{1}\right) K_{\bar{N}}\left(u_{N}\right) R_{1, \bar{N}}\left(u_{N}+\xi_{1}\right) \ldots R_{N, \bar{N}}\left(u_{N}+\xi_{N}\right) \\
& \quad \times \phi_{\lambda, \lambda-\eta \hat{2}}^{1}\left(\xi_{1}\right) \ldots \phi_{\lambda-(N-1) \eta \hat{2}, \lambda-N \eta \hat{2}}^{N}\left(\xi_{N}\right) \phi_{\lambda-(N-2) \eta \hat{1}, \lambda-(N-1) \eta \hat{1}}^{\overline{1}}\left(-u_{1}\right) \ldots \phi_{\lambda+N \eta \hat{1}, \lambda+(N-1) \eta \hat{1}}^{\bar{N}}\left(-u_{N}\right) .
\end{aligned}
$$




\section{Partition function in terms of the face type mon- odromy matrix}

Let us introduce the face type one-row monodromy matrix

$$
\begin{aligned}
T_{F}(l \mid u) & \equiv T_{0,1 \ldots N}^{F}(l \mid u) \\
& =R_{0, N}\left(u-\xi_{N} ; l-\eta \sum_{i=1}^{N-1} h^{(i)}\right) \ldots R_{0,2}\left(u-\xi_{2} ; l-\eta h^{(1)}\right) R_{0,1}\left(u-\xi_{1} ; l\right) \\
& =\left(\begin{array}{ll}
T_{F}(l \mid u)_{1}^{1} & T_{F}(l \mid u)_{2}^{1} \\
T_{F}(l \mid u)_{1}^{2} & T_{F}(l \mid u)_{2}^{2}
\end{array}\right)
\end{aligned}
$$

where $l$ is a generic vector in $V$. The monodromy matrix satisfies the face type quadratic exchange relation [49, 50]. Applying $T_{F}(l \mid u)_{j}^{i}$ to an arbitrary vector $\left|i_{1}, \ldots, i_{N}\right\rangle$ in the Ntensor product space $V^{\otimes N}$ given by

$$
\left|i_{1}, \ldots, i_{N}\right\rangle=\epsilon_{i_{1}}^{1} \ldots \epsilon_{i_{N}}^{N}
$$

we have

$$
\begin{aligned}
T_{F}(l \mid u)_{j}^{i}\left|i_{1}, \ldots, i_{N}\right\rangle \equiv & T_{F}(m ; l \mid u)_{j}^{i}\left|i_{1}, \ldots, i_{N}\right\rangle \\
= & \sum_{\alpha_{N-1} \ldots \alpha_{1}} \sum_{i_{N}^{\prime} \ldots i_{1}^{\prime}} R\left(u-\xi_{N} ; l-\eta \sum_{k=1}^{N-1} \hat{\imath}_{k}^{\prime}\right)_{\alpha_{N-1}}^{i} i_{N}^{i_{N}^{\prime}} \ldots \\
& \quad \times R\left(u-\xi_{2} ; l-\eta \hat{\imath}_{1}^{\prime}\right)_{\alpha_{1}}^{\alpha_{2} i_{2}^{\prime}} R\left(u-\xi_{1} ; l\right)_{j}^{\alpha_{1} i_{1}^{\prime}}\left|i_{1}^{\prime}, \ldots, i_{N}^{\prime}\right\rangle,
\end{aligned}
$$

where $m=l-\eta \sum_{k=1}^{N} \hat{\imath}_{k}$.

Now we compute the partition function $Z_{N}\left(\left\{u_{\alpha}\right\} ;\left\{\xi_{i}\right\} ; \lambda ; \zeta\right)$ (2.13). The expression (2.42) implies that

$$
\begin{aligned}
Z_{N}\left(\left\{u_{\alpha}\right\} ;\left\{\xi_{i}\right\} ; \lambda ; \zeta\right)=\left\langle\Omega^{(1)}(\lambda)\right| \tilde{\phi}_{\lambda-(N-1) \eta \hat{1}+\eta \hat{2}, \lambda-(N-1) \eta \hat{1}}^{\overline{1}}\left(u_{1}\right) \mathbb{T}_{\overline{1}}\left(u_{1}\right) \phi_{\lambda-(N-2) \eta \hat{1}, \lambda-(N-1) \eta \hat{1}}^{\overline{1}}\left(-u_{1}\right) \\
\vdots \\
\quad \times \tilde{\phi}_{\lambda+(N-1) \eta \hat{1}+\eta \hat{2}, \lambda+(N-1) \eta \hat{1}}^{\bar{N}}\left(u_{N}\right) \mathbb{T}_{\bar{N}}\left(u_{N}\right) \phi_{\lambda+N \eta \hat{1}, \lambda+(N-1) \eta \hat{1}}^{\bar{N}}\left(-u_{N}\right)\left|\Omega^{(2)}(\lambda)\right\rangle .
\end{aligned}
$$

With the help of the crossing relation (2.27), the face-vertex correspondence relation (2.32) and the relations $(2.33)$, following the procedure in [28, 51], we find that the partition function $Z_{N}\left(\left\{u_{\alpha}\right\} ;\left\{\xi_{i}\right\} ; \lambda ; \zeta\right)$ can be expressed in terms of the face-type double-row monodromy 
operators

$$
\begin{gathered}
Z_{N}\left(\left\{u_{\alpha}\right\} ;\left\{\xi_{i}\right\} ; \lambda ; \zeta\right)=\langle 1, \ldots, 1| \mathcal{T}_{F}^{-}\left(\lambda-2(M-1) \eta \hat{1}, \lambda \mid u_{1}\right)_{1}^{2} \ldots \mathcal{T}_{F}^{-}\left(\lambda, \lambda \mid u_{M}\right)_{1}^{2} \\
\times \mathcal{T}_{F}^{-}\left(\lambda+2 \eta \hat{1}, \lambda \mid u_{M+1}\right)_{1}^{2} \ldots \mathcal{T}_{F}^{-}\left(\lambda+N \eta \hat{1}, \lambda \mid u_{N}\right)_{1}^{2}|2, \ldots, 2\rangle
\end{gathered}
$$

The above double-row monodromy matrix operator $\mathcal{T}_{F}^{-}(m, \lambda \mid u)_{1}^{2}$ is given by the one-row monodromy matrix operator $T_{F}(m ; l \mid u)_{j}^{i}[52$

$$
\begin{aligned}
& \mathcal{T}_{F}^{-}(m, \lambda \mid u)_{1}^{2}=\frac{\sigma\left(m_{21}\right)}{\sigma\left(\lambda_{21}\right)} \prod_{k=1}^{N} \frac{\sigma\left(u+\xi_{k}\right)}{\sigma\left(u+\xi_{k}+\eta\right)} \\
& \quad \times\left\{\frac{\sigma\left(\lambda_{1}+\zeta-u\right)}{\sigma\left(\lambda_{1}+\zeta+u\right)} T_{F}(m, \lambda \mid u)_{1}^{2} T_{F}(m+\eta \hat{2}, \lambda+\eta \hat{2} \mid-u-\eta)_{2}^{2}\right. \\
& \left.\quad-\frac{\sigma\left(\lambda_{2}+\zeta-u\right)}{\sigma\left(\lambda_{2}+\zeta+u\right)} T_{F}(m+2 \eta \hat{2}, \lambda \mid u)_{2}^{2} T_{F}(m+\eta \hat{1}, \lambda+\eta \hat{1} \mid-u-\eta)_{1}^{2}\right\} .
\end{aligned}
$$

\section{$4 \quad$ F-basis}

In this section, after briefly reviewing the result [53] about the Drinfeld twist [21] (factorizing F-matrix) of the eight-vertex SOS model, we obtain the explicit expression of the double rows monodromy operator $\mathcal{T}_{F}^{-}(m, \lambda \mid u)_{1}^{2}$ given by (3.6) in the F-basis provided by the F-matrix.

\subsection{Factorizing Drinfeld twist $F$}

Let $\mathcal{S}_{N}$ be the permutation group over indices $1, \ldots, N$ and $\left\{s_{i} \mid i=1, \ldots, N-1\right\}$ be the set

of elementary permutations in $\mathcal{S}_{N}$. For each elementary permutation $s_{i}$, we introduce the associated operator $R_{1 \ldots N}^{s_{i}}$ on the quantum space

$$
R_{1 \ldots N}^{s_{i}}(l) \equiv R^{s_{i}}(l)=R_{i, i+1}\left(\xi_{i}-\xi_{i+1} \mid l-\eta \sum_{k=1}^{i-1} h^{(k)}\right),
$$

where $l$ is a generic vector in $V$. For any $s, s^{\prime} \in \mathcal{S}_{N}$, operator $R_{1 \ldots N}^{s s^{\prime}}$ associated with $s s^{\prime}$ satisfies the following composition law:

$$
R_{1 \ldots N}^{s s^{\prime}}(l)=R_{s(1 \ldots N)}^{s^{\prime}}(l) R_{1 \ldots N}^{s}(l)
$$

Let $s$ be decomposed in a minimal way in terms of elementary permutations,

$$
s=s_{\beta_{1}} \ldots s_{\beta_{p}}
$$


where $\beta_{i}=1, \ldots, N-1$ and the positive integer $p$ is the length of $s$. The composition law (4.2) enables one to obtain operator $R_{1 \ldots N}^{s}$ associated with each $s \in \mathcal{S}_{N}$. The dynamical quantum Yang-Baxter equation (2.23), weight conservation condition (2.25) and unitary condition (2.26) guarantee the uniqueness of $R_{1 \ldots N}^{s}$. Moreover, one may check that $R_{1 \ldots N}^{s}$ satisfies the following exchange relation with the face type one-row monodromy matrix (3.1)

$$
R_{1 \ldots N}^{s}(l) T_{0,1 \ldots N}^{F}(l \mid u)=T_{0, s(1 \ldots N)}^{F}(l \mid u) R_{1 \ldots N}^{s}\left(l-\eta h^{(0)}\right), \quad \forall s \in \mathcal{S}_{N}
$$

Now, we construct the face-type Drinfeld twist $F_{1 \ldots N}(l) \equiv F_{1 \ldots N}\left(l ; \xi_{1}, \ldots, \xi_{N}\right) 2$ on the $N$-fold tensor product space $V^{\otimes N}$, which satisfies the following three properties:

$$
\begin{aligned}
& \text { I. lower - triangularity; } \\
& \text { II. non - degeneracy; } \\
& \text { III. factorizing property : } R_{1 \ldots N}^{s}(l)=F_{s(1 \ldots N)}^{-1}(l) F_{1 \ldots N}(l), \forall s \in \mathcal{S}_{N} \text {. }
\end{aligned}
$$

Substituting (4.7) into the exchange relation (4.4) yields the following relation

$$
F_{s(1 \ldots N)}^{-1}(l) F_{1 \ldots N}(l) T_{0,1 \ldots N}^{F}(l \mid u)=T_{0, s(1 \ldots N)}^{F}(l \mid u) F_{s(1 \ldots N)}^{-1}\left(l-\eta h^{(0)}\right) F_{1 \ldots N}\left(l-\eta h^{(0)}\right) .
$$

Equivalently,

$$
F_{1 \ldots N}(l) T_{0,1 \ldots N}^{F}(l \mid u) F_{1 \ldots N}^{-1}\left(l-\eta h^{(0)}\right)=F_{s(1 \ldots N)}(l) T_{0, s(1 \ldots N)}^{F}(l \mid u) F_{s(1 \ldots N)}^{-1}\left(l-\eta h^{(0)}\right) .
$$

Let us introduce the twisted monodromy matrix $\tilde{T}_{0,1 \ldots N}^{F}(l \mid u)$ by

$$
\begin{aligned}
\tilde{T}_{0,1 \ldots N}^{F}(l \mid u) & =F_{1 \ldots N}(l) T_{0,1 \ldots N}^{F}(l \mid u) F_{1 \ldots N}^{-1}\left(l-\eta h^{(0)}\right) \\
& =\left(\begin{array}{ll}
\tilde{T}_{F}(l \mid u)_{1}^{1} & \tilde{T}_{F}(l \mid u)_{2}^{1} \\
\tilde{T}_{F}(l \mid u)_{1}^{2} & \tilde{T}_{F}(l \mid u)_{2}^{2}
\end{array}\right) .
\end{aligned}
$$

Then (4.9) implies that the twisted monodromy matrix is symmetric under $\mathcal{S}_{N}$, namely,

$$
\tilde{T}_{0,1 \ldots N}^{F}(l \mid u)=\tilde{T}_{0, s(1 \ldots N)}^{F}(l \mid u), \quad \forall s \in \mathcal{S}_{N}
$$

Define the F-matrix:

$$
F_{1 \ldots N}(l)=\sum_{s \in \mathcal{S}_{N}} \sum_{\left\{\alpha_{j}\right\}=1}^{2}{ }^{*} \prod_{j=1}^{N} P_{\alpha_{s(j)}(j)} R_{1 \ldots N}^{s}(l),
$$

\footnotetext{
${ }^{2}$ In this paper, we adopt the convention: $F_{s(1 \ldots N)}(l) \equiv F_{s(1 \ldots N)}\left(l ; \xi_{s(1)}, \ldots, \xi_{s(N)}\right)$.
} 
where $P_{\alpha}^{i}$ is the embedding of the project operator $P_{\alpha}$ in the $i^{\text {th }}$ space with matrix elements $\left(P_{\alpha}\right)_{k l}=\delta_{k l} \delta_{k \alpha}$. The sum $\sum^{*}$ in (4.12) is over all non-decreasing sequences of the labels $\alpha_{s(i)}$ :

$$
\begin{array}{ll}
\alpha_{s(i+1)} \geq \alpha_{s(i)} & \text { if } \quad s(i+1)>s(i), \\
\alpha_{s(i+1)}>\alpha_{s(i)} & \text { if } \quad s(i+1)<s(i) .
\end{array}
$$

From (4.13),$F_{1 \ldots N}(l)$ obviously is a lower-triangular matrix. Moreover, the F-matrix is nondegenerate because all its diagonal elements are non-zero. It was shown in [53] that the F-matrix also satisfies the factorizing property (4.7).

\subsection{Completely symmetric representations}

In the F-basis provided by the F-matrix (4.12), the twisted operators $\tilde{T}_{F}(l \mid u)_{i}^{j}$ defined by (4.10) become polarization free [53, 54]. Here we present the results relevant for our purpose

$$
\begin{aligned}
& \tilde{T}_{F}(l \mid u)_{2}^{2}=\frac{\sigma\left(l_{21}-\eta\right)}{\sigma\left(l_{21}-\eta+\eta\left\langle H, \epsilon_{1}\right\rangle\right)} \otimes_{i}\left(\begin{array}{cc}
\frac{\sigma\left(u-\xi_{i}\right)}{\sigma\left(u-\xi_{i}+\eta\right)} & \\
& 1
\end{array}\right)_{(i)}, \\
& \tilde{T}_{F}(l \mid u)_{1}^{2}=\sum_{i=1}^{N} \frac{\sigma(\eta) \sigma\left(u-\xi_{i}+l_{12}\right)}{\sigma\left(u-\xi_{i}+\eta\right) \sigma\left(l_{12}\right)} E_{12}^{i} \otimes_{j \neq i}\left(\begin{array}{cc}
\frac{\sigma\left(u-\xi_{j}\right) \sigma\left(\xi_{i}-\xi_{j}+\eta\right)}{\sigma\left(u-\xi_{j}+\eta\right) \sigma\left(\xi_{i}-\xi_{j}\right)} & \\
& 1
\end{array}\right)_{(j)} .
\end{aligned}
$$

Applying the above operators to the arbitrary state $\left|i_{1}, \ldots, i_{N}\right\rangle$ given by (3.2) leads to

$$
\begin{aligned}
\tilde{T}_{F}(m, l \mid u)_{2}^{2}= & \frac{\sigma\left(l_{21}-\eta\right)}{\sigma\left(l_{2}-m_{1}-\eta\right)} \otimes_{i}\left(\begin{array}{ll}
\frac{\sigma\left(u-\xi_{i}\right)}{\sigma\left(u-\xi_{i}+\eta\right)} & \\
& 1
\end{array}\right)_{(i)}, \\
\tilde{T}_{F}(m, l \mid u)_{1}^{2}= & \sum_{i=1}^{N} \frac{\sigma(\eta) \sigma\left(u-\xi_{i}+l_{12}\right)}{\sigma\left(u-\xi_{i}+\eta\right) \sigma\left(l_{12}\right)} \\
& \times E_{12}^{i} \otimes_{j \neq i}\left(\begin{array}{cc}
\frac{\sigma\left(u-\xi_{j}\right) \sigma\left(\xi_{i}-\xi_{j}+\eta\right)}{\sigma\left(u-\xi_{j}+\eta\right) \sigma\left(\xi_{i}-\xi_{j}\right)} & \\
& 1
\end{array}\right)_{(j)} .
\end{aligned}
$$

It then follows that the pseudo-particle creation operator (3.6) in the F-basis has the following completely symmetric polarization free form:

$$
\begin{aligned}
& \tilde{\mathcal{T}}_{F}^{-}(m, \lambda \mid u)_{1}^{2}=\frac{\sigma\left(m_{12}\right)}{\sigma\left(m_{1}-\lambda_{2}\right)} \prod_{k=1}^{N} \frac{\sigma\left(u+\xi_{k}\right)}{\sigma\left(u+\xi_{k}+\eta\right)} \\
& \quad \times \sum_{i=1}^{N} \frac{\sigma\left(\lambda_{1}+\zeta-\xi_{i}\right) \sigma\left(\lambda_{2}+\zeta+\xi_{i}\right) \sigma(2 u) \sigma(\eta)}{\sigma\left(\lambda_{1}+\zeta+u\right) \sigma\left(\lambda_{2}+\zeta+u\right) \sigma\left(u-\xi_{i}+\eta\right) \sigma\left(u+\xi_{i}\right)}
\end{aligned}
$$




$$
\times E_{12}^{i} \otimes_{j \neq i}\left(\begin{array}{cc}
\frac{\sigma\left(u-\xi_{j}\right) \sigma\left(u+\xi_{j}+\eta\right) \sigma\left(\xi_{i}-\xi_{j}+\eta\right)}{\sigma\left(u-\xi_{j}+\eta\right) \sigma\left(u+\xi_{j}\right) \sigma\left(\xi_{i}-\xi_{j}\right)} & \\
& 1
\end{array}\right)_{(j)}
$$

\section{Determinant representation of the partition function}

In this section we compute the DW partition function $Z_{N}\left(\left\{u_{\alpha}\right\} ;\left\{\xi_{i}\right\} ; \lambda ; \zeta\right)$ (3.5) using the expansion of the twisted operator $\tilde{\mathcal{T}}_{F}^{-}(m, \lambda \mid u)_{1}^{2}(4.18)$.

\subsection{Symmetric expression of the partition function}

From the definition of the F-matrix $F_{1 \ldots N}(l)(4.12)$, we can show that the state $|2, \ldots, 2\rangle$ and the dual state $\langle 1, \ldots, 1|$ are invariant under the action of $F_{1 \ldots N}(l)$, namely,

$$
\begin{aligned}
F_{1 \ldots N}(l)|2, \ldots, 2\rangle & =|2, \ldots, 2\rangle \\
\langle 1, \ldots, 1| F_{1 \ldots N}(l) & =\langle 1, \ldots, 1| .
\end{aligned}
$$

Hence the DW partition function $Z_{N}\left(\left\{u_{\alpha}\right\} ;\left\{\xi_{j}\right\} ; \lambda ; \zeta\right)$ can be expressed in terms of the twisted operator $\tilde{\mathcal{T}}_{F}^{-}(m, \lambda \mid u)_{1}^{2}$ as follow

$$
\begin{aligned}
& Z_{N}\left(\left\{u_{\alpha}\right\} ;\left\{\xi_{i}\right\} ; \lambda ; \zeta\right)=\left\langle 1, \ldots, 1\left|\mathcal{T}_{F}^{-}\left(\lambda-(N-2) \eta \hat{1}, \lambda \mid u_{1}\right)_{1}^{2} \ldots \mathcal{T}_{F}^{-}\left(\lambda+N \eta \hat{1}, \lambda \mid u_{N}\right)_{1}^{2}\right| 2, \ldots, 2\right\rangle \\
&=\langle 1, \ldots, 1| F_{1 \ldots N}(\lambda-N \eta \hat{1}) \mathcal{T}_{F}^{-}\left(\lambda-(N-2) \eta \hat{1}, \lambda \mid u_{1}\right)_{1}^{2} \ldots \\
& \quad \times \mathcal{T}_{F}^{-}\left(\lambda+N \eta \hat{1}, \lambda \mid u_{N}\right)_{1}^{2} F_{1 \ldots N}^{-1}(\lambda+N \eta \hat{1})|2, \ldots, 2\rangle \\
&=\left\langle 1, \ldots, 1\left|\tilde{\mathcal{T}}_{F}^{-}\left(\lambda-(N-2) \eta \hat{1}, \lambda \mid u_{1}\right)_{1}^{2} \ldots \tilde{\mathcal{T}}_{F}^{-}\left(\lambda+N \eta \hat{1}, \lambda \mid u_{N}\right)_{1}^{2}\right| 2, \ldots, 2\right\rangle .
\end{aligned}
$$

Substituting the polarization free expression (4.18) of the twisted operator $\tilde{\mathcal{T}}_{F}^{-}(m, \lambda \mid u)_{1}^{2}$ into the above equation, we have

$$
\begin{aligned}
& Z_{N}\left(\left\{u_{\alpha}\right\} ;\left\{\xi_{i}\right\} ; \lambda ; \zeta\right)=\prod_{k=1}^{M} \frac{\sigma\left(\lambda_{12}+2 k \eta\right) \sigma\left(\lambda_{12}-2 k \eta+\eta\right)}{\sigma\left(\lambda_{12}+k \eta\right) \sigma\left(\lambda_{12}-k \eta+\eta\right)} \prod_{l=1}^{N} \prod_{i=1}^{N} \frac{\sigma\left(u_{i}+\xi_{l}\right)}{\sigma\left(u_{i}+\xi_{l}+\eta\right)}\langle 1, \ldots, 1| \\
& \times \sum_{i=1}^{N} \frac{\sigma\left(\lambda_{1}+\zeta-\xi_{i}\right) \sigma\left(\lambda_{2}+\zeta+\xi_{i}\right) \sigma\left(2 u_{1}\right) \sigma(\eta)}{\sigma\left(\lambda_{1}+\zeta+u_{1}\right) \sigma\left(\lambda_{2}+\zeta+u_{1}\right) \sigma\left(u_{1}-\xi_{i}+\eta\right) \sigma\left(u_{1}+\xi_{i}\right)} E_{12}^{i} \otimes_{j \neq i}\left(\frac{\sigma\left(u_{1}-\xi_{j}\right) \sigma\left(u_{1}+\xi_{j}+\eta\right) \sigma\left(\xi_{i} \xi_{j}+\eta\right)}{\sigma\left(u_{1}-\xi_{j}+\eta\right) \sigma\left(u_{1}+\xi_{j}\right) \sigma\left(\xi_{i}-\xi_{j}\right)}\right)_{(j)} \\
& \quad \vdots \\
& \times \sum_{i=1}^{N} \frac{\sigma\left(\lambda_{1}+\zeta-\xi_{i}\right) \sigma\left(\lambda_{2}+\zeta+\xi_{i}\right) \sigma\left(2 u_{N}\right) \sigma(\eta)}{\sigma\left(\lambda_{1}+\zeta+u_{N}\right) \sigma\left(\lambda_{2}+\zeta+u_{N}\right) \sigma\left(u_{N}-\xi_{i}+\eta\right) \sigma\left(u_{N}+\xi_{i}\right)} E_{12}^{i} \otimes_{j \neq i}\left(\frac{\sigma\left(u_{N}-\xi_{j}\right) \sigma\left(u_{N}+\xi_{j}+\eta\right) \sigma\left(\xi_{i}-\xi_{j}+\eta\right)}{\sigma\left(u_{N}-\xi_{j}+\eta\right) \sigma\left(u_{N}+\xi_{j}\right) \sigma\left(\xi_{i}-\xi_{j}\right)}\right. \\
& \quad \times|2, \ldots, 2\rangle .
\end{aligned}
$$


Expanding the sum in the above equation corresponding to the spectral parameter $u_{N}$ yields

$$
\begin{aligned}
& Z_{N}\left(\left\{u_{\alpha}\right\} ;\left\{\xi_{i}\right\} ; \lambda ; \zeta\right)=\prod_{k=1}^{M} \frac{\sigma\left(\lambda_{12}+2 k \eta\right) \sigma\left(\lambda_{12}-2 k \eta+\eta\right)}{\sigma\left(\lambda_{12}+k \eta\right) \sigma\left(\lambda_{12}-k \eta+\eta\right)} \prod_{l=1}^{N} \prod_{i=1}^{N} \frac{\sigma\left(u_{i}+\xi_{l}\right)}{\sigma\left(u_{i}+\xi_{l}+\eta\right)} \\
& \times \sum_{i=1}^{N} \frac{\sigma\left(\lambda_{1}+\zeta-\xi_{i}\right) \sigma\left(\lambda_{2}+\zeta+\xi_{i}\right) \sigma\left(2 u_{N}\right) \sigma(\eta)}{\sigma\left(\lambda_{1}+\zeta+u_{N}\right) \sigma\left(\lambda_{2}+\zeta+u_{N}\right) \sigma\left(u_{N}-\xi_{i}+\eta\right) \sigma\left(u_{N}+\xi_{i}\right)} \prod_{l=1}^{N-1} \frac{\sigma\left(u_{l}-\xi_{i}\right) \sigma\left(u_{l}+\xi_{i}+\eta\right)}{\sigma\left(u_{l}-\xi_{i}+\eta\right) \sigma\left(u_{l}+\xi_{i}\right)} \\
& \times \prod_{j \neq i} \frac{\sigma\left(\xi_{j}-\xi_{i}+\eta\right)}{\sigma\left(\xi_{j}-\xi_{i}\right)}\langle 1, \ldots, 1| \\
& \times \sum_{l \neq i}^{N} \frac{\sigma\left(\lambda_{1}+\zeta-\xi_{l}\right) \sigma\left(\lambda_{2}+\zeta+\xi_{l}\right) \sigma\left(2 u_{1}\right) \sigma(\eta)}{\sigma\left(\lambda_{1}+\zeta+u_{1}\right) \sigma\left(\lambda_{2}+\zeta+u_{1}\right) \sigma\left(u_{1}-\xi_{l}+\eta\right) \sigma\left(u_{1}+\xi_{l}\right)} E_{12}^{i} \otimes_{j \neq l, i}\left(\frac{\sigma\left(u_{1}-\xi_{j}\right) \sigma\left(u_{1}+\xi_{j}+\eta\right) \sigma\left(\xi_{l}-\xi_{j}+\eta\right)}{\sigma\left(u_{1} \xi_{j}+\eta\right) \sigma\left(u_{1}+\xi_{j}\right) \sigma\left(\xi_{l} \xi_{j}\right)} \quad 1\right)_{(j)} \\
& \times|2, \ldots, 2\rangle .
\end{aligned}
$$

Iterating the above procedure, we obtain the complete symmetric expression of the partition function $Z_{N}\left(\left\{u_{\alpha}\right\} ;\left\{\xi_{i}\right\} ; \lambda ; \zeta\right)$

$$
\begin{aligned}
Z_{N}\left(\left\{u_{\alpha}\right\} ;\left\{\xi_{i}\right\} ; \lambda ; \zeta\right)=\prod_{k=1}^{M} & \frac{\sigma\left(\lambda_{12}+2 k \eta\right) \sigma\left(\lambda_{12}-2 k \eta+\eta\right)}{\sigma\left(\lambda_{12}+k \eta\right) \sigma\left(\lambda_{12}-k \eta+\eta\right)} \prod_{l=1}^{N} \prod_{i=1}^{N} \frac{\sigma\left(u_{i}+\xi_{l}\right)}{\sigma\left(u_{i}+\xi_{l}+\eta\right)} \\
& \times \mathcal{Z}_{N}\left(\left\{u_{\alpha}\right\} ;\left\{\xi_{i}\right\} ; \lambda ; \zeta\right),
\end{aligned}
$$

where the normalized partition function $\mathcal{Z}_{N}\left(\left\{u_{\alpha}\right\} ;\left\{\xi_{i}\right\} ; \lambda ; \zeta\right)$ is

$$
\begin{aligned}
\mathcal{Z}_{N}\left(\left\{u_{\alpha}\right\} ;\left\{\xi_{i}\right\} ; \lambda ; \zeta\right)= & \sum_{s \in \mathcal{S}_{N}} \prod_{n=1}^{N}\left\{\frac{\sigma\left(\lambda_{1}+\zeta-\xi_{i_{s(n)}}\right) \sigma\left(\lambda_{2}+\zeta+\xi_{i_{s(n)}}\right) \sigma\left(2 u_{n}\right) \sigma(\eta)}{\sigma\left(\lambda_{1}+\zeta+u_{n}\right) \sigma\left(\lambda_{2}+\zeta+u_{n}\right) \sigma\left(u_{n}-\xi_{i_{s(n)}}+\eta\right) \sigma\left(u_{n}+\xi_{i_{s(n)}}\right)}\right. \\
& \left.\times \prod_{k>n}^{N} \frac{\sigma\left(u_{n}-\xi_{i_{s(k)}}\right) \sigma\left(u_{n}+\xi_{i_{s(k)}}+\eta\right) \sigma\left(\xi_{i_{s(n)}}-\xi_{i_{s(k)}}+\eta\right)}{\sigma\left(u_{n}-\xi_{i_{s(k)}}+\eta\right) \sigma\left(u_{n}+\xi_{i_{s(k)}}\right) \sigma\left(\xi_{i_{s(n)}}-\xi_{\left.i_{s(k)}\right)}\right)}\right\}
\end{aligned}
$$

\subsection{Recursive relation and the determinant representation}

From the expression (5.4), it is easy to check that the partition function $\mathcal{Z}_{N}\left(\left\{u_{\alpha}\right\} ;\left\{\xi_{i}\right\} ; \lambda ; \zeta\right)$

is a symmetric function of $\left\{u_{\alpha}\right\}$ and $\left\{\xi_{i}\right\}$ separatively. Moreover, we can show that the partition function $\mathcal{Z}_{N}\left(\left\{u_{\alpha}\right\} ;\left\{\xi_{i}\right\} ; \lambda ; \zeta\right)$ satisfy the following recursive relation

$$
\mathcal{Z}_{N}\left(\left\{u_{\alpha}\right\} ;\left\{\xi_{i}\right\} ; \lambda ; \zeta\right)=\sum_{i=1}^{N} \frac{\sigma\left(\lambda_{1}+\zeta-\xi_{i}\right) \sigma\left(\lambda_{2}+\zeta+\xi_{i}\right) \sigma\left(2 u_{N}\right) \sigma(\eta)}{\sigma\left(\lambda_{1}+\zeta+u_{N}\right) \sigma\left(\lambda_{2}+\zeta+u_{N}\right) \sigma\left(u_{N}-\xi_{i}+\eta\right) \sigma\left(u_{N}+\xi_{i}\right)}
$$




$$
\begin{aligned}
& \times \prod_{l=1}^{N-1} \frac{\sigma\left(u_{l}-\xi_{i}\right) \sigma\left(u_{l}+\xi_{i}+\eta\right)}{\sigma\left(u_{l}-\xi_{i}+\eta\right) \sigma\left(u_{l}+\xi_{i}\right)} \prod_{j \neq i} \frac{\sigma\left(\xi_{j}-\xi_{i}+\eta\right)}{\sigma\left(\xi_{j}-\xi_{i}\right)} \\
& \times \mathcal{Z}_{N-1}\left(\left\{u_{\alpha}\right\}_{\alpha \neq N} ;\left\{\xi_{j}\right\}_{j \neq i} ; \lambda ; \zeta\right) .
\end{aligned}
$$

One can show that the initial condition $\mathcal{Z}_{0}\left(\left\{u_{\alpha}\right\} ;\left\{\xi_{i}\right\} ; \lambda ; \zeta\right)=1$ and the recursive relation (5.5) uniquely determinate the partition function $\mathcal{Z}_{N}\left(\left\{u_{\alpha}\right\} ;\left\{\xi_{i}\right\} ; \lambda ; \zeta\right)$ for any positive integer $N$. This fact allows us to obtain the following determinant representation of the normalized partition function $\mathcal{Z}_{N}\left(\left\{u_{\alpha}\right\} ;\left\{\xi_{i}\right\} ; \lambda ; \zeta\right)$ :

$$
\mathcal{Z}_{N}\left(\left\{u_{\alpha}\right\} ;\left\{\xi_{i}\right\} ; \lambda ; \zeta\right)=\frac{\prod_{\alpha=1}^{N} \prod_{i=1}^{N} \sigma\left(u_{\alpha}-\xi_{i}\right) \sigma\left(u_{\alpha}+\xi_{i}+\eta\right) \operatorname{det} \mathcal{N}\left(\left\{u_{\alpha}\right\} ;\left\{\xi_{i}\right\}\right)}{\prod_{\alpha>\beta} \sigma\left(u_{\alpha}-u_{\beta}\right) \sigma\left(u_{\alpha}+u_{\beta}+\eta\right) \prod_{k<l} \sigma\left(\xi_{k}-\xi_{l}\right) \sigma\left(\xi_{k}+\xi_{l}\right)},
$$

where the $N \times N$ matrix $\mathcal{N}\left(\left\{u_{\alpha}\right\} ;\left\{\xi_{i}\right\}\right)$ is given by

$$
\begin{aligned}
\mathcal{N}\left(\left\{u_{\alpha}\right\} ;\left\{\xi_{i}\right\}\right)_{\alpha, j}= & \frac{\sigma(\eta) \sigma\left(\lambda_{1}+\zeta-\xi_{j}\right)}{\sigma\left(u_{\alpha}-\xi_{j}\right) \sigma\left(u_{\alpha}+\xi_{j}+\eta\right) \sigma\left(\lambda_{1}+\zeta+u_{\alpha}\right)} \\
& \times \frac{\sigma\left(\lambda_{2}+\zeta+\xi_{j}\right) \sigma\left(2 u_{\alpha}\right)}{\sigma\left(\lambda_{2}+\zeta+u_{\alpha}\right) \sigma\left(u_{\alpha}-\xi_{j}+\eta\right) \sigma\left(u_{\alpha}+\xi_{j}\right)} .
\end{aligned}
$$

We shall prove the above determinant representation as follows. Firstly let us introduce two sets of functions $\left\{B_{I}\left(\left\{u_{\alpha}\right\} ;\left\{\xi_{i}\right\} ; \lambda ; \zeta\right) \mid I=1, \ldots, N\right\}$ and $\left\{F_{I}\left(\left\{u_{\alpha}\right\} ;\left\{\xi_{i}\right\} ; \lambda ; \zeta\right) \mid I=\right.$ $1, \ldots, N\}$ defined respectively by

$$
\begin{aligned}
B_{I}\left(\left\{u_{\alpha}\right\} ;\left\{\xi_{i}\right\} ; \lambda ; \zeta\right)= & \prod_{l=1}^{I} \frac{\sigma\left(\lambda_{1}+\zeta+u_{l}\right) \sigma\left(\lambda_{2}+\zeta+u_{l}\right)}{\sigma\left(\lambda_{1}+\zeta-\xi_{l}\right) \sigma\left(\lambda_{2}+\zeta+\xi_{l}\right) \sigma\left(2 u_{l}\right)} \mathcal{Z}_{I}\left(\left\{u_{\alpha}\right\} ;\left\{\xi_{i}\right\} ; \lambda ; \zeta\right), \\
F_{I}\left(\left\{u_{\alpha}\right\} ;\left\{\xi_{i}\right\} ; \lambda ; \zeta\right)= & \frac{\prod_{\alpha=1}^{I} \prod_{j=1}^{I} \sigma\left(u_{\alpha}-\xi_{j}\right) \sigma\left(u_{\alpha}+\xi_{j}+\eta\right)}{\prod_{\alpha>\beta} \sigma\left(u_{\alpha}-u_{\beta}\right) \sigma\left(u_{\alpha}+u_{\beta}+\eta\right) \prod_{k<l} \sigma\left(\xi_{k}-\xi_{l}\right) \sigma\left(\xi_{k}+\xi_{l}\right)} \\
& \times \operatorname{det}\left|\frac{\sigma(\eta)}{\sigma\left(u_{\alpha}-\xi_{j}\right) \sigma\left(u_{\alpha}+\xi_{j}+\eta\right) \sigma\left(u_{\alpha}-\xi_{j}+\eta\right) \sigma\left(u_{\alpha}+\xi_{j}\right)}\right| .
\end{aligned}
$$

Then the proof of (5.6) is equivalent to proving the equation

$$
B_{I}\left(\left\{u_{\alpha}\right\} ;\left\{\xi_{i}\right\} ; \lambda ; \zeta\right)=F_{I}\left(\left\{u_{\alpha}\right\} ;\left\{\xi_{i}\right\} ; \lambda ; \zeta\right), \quad \text { for any positive integer I. }
$$

We prove (5.10) by induction.

- From direct calculation, we can show that (5.10) holds for the case of $I=1$, namely,

$$
B_{1}\left(u_{1} ; \xi_{1} ; \lambda ; \zeta\right)=F_{1}\left(u_{1} ; \xi_{1} ; \lambda ; \zeta\right)=\frac{\sigma(\eta)}{\sigma\left(u_{1}-\xi_{1}+\eta\right) \sigma\left(u_{1}+\xi_{1}\right)} .
$$


- Suppose that (5.10) holds for the case of $I \leq N-1$. We now prove that it is satisfied also for $I=N$. It is easy to check that both $B_{N}\left(\left\{u_{\alpha}\right\} ;\left\{\xi_{i}\right\} ; \lambda ; \zeta\right)$ and $F_{N}\left(\left\{u_{\alpha}\right\} ;\left\{\xi_{i}\right\} ; \lambda ; \zeta\right)$ are symmetric functions of $\left\{u_{\alpha}\right\}$. Hence it is sufficient to prove that they are equal as functions of $u_{N}$. The recursive relation (5.5) of $\mathcal{Z}_{N}\left(\left\{u_{\alpha}\right\} ;\left\{\xi_{i}\right\} ; \lambda ; \zeta\right)$ implies that $B_{N}\left(\left\{u_{\alpha}\right\} ;\left\{\xi_{i}\right\} ; \lambda ; \zeta\right)$ satisfies the following relation

$$
\begin{aligned}
B_{N}\left(\left\{u_{\alpha}\right\} ;\left\{\xi_{i}\right\} ; \lambda ; \zeta\right)= & \sum_{i=1}^{N} \frac{\sigma(\eta)}{\sigma\left(u_{N}-\xi_{i}+\eta\right) \sigma\left(u_{N}+\xi_{i}\right)} \prod_{l=1}^{N-1} \frac{\sigma\left(u_{l}-\xi_{i}\right) \sigma\left(u_{l}+\xi_{i}+\eta\right)}{\sigma\left(u_{l}-\xi_{i}+\eta\right) \sigma\left(u_{l}+\xi_{i}\right)} \\
& \times \prod_{j \neq i} \frac{\sigma\left(\xi_{j}-\xi_{i}+\eta\right)}{\sigma\left(\xi_{j}-\xi_{i}\right)} B_{N-1}\left(\left\{u_{\alpha}\right\}_{\alpha \neq N} ;\left\{\xi_{j}\right\}_{j \neq i} ; \lambda ; \zeta\right) .
\end{aligned}
$$

The determinant representation of the function $F_{N}\left(\left\{u_{\alpha}\right\} ;\left\{\xi_{i}\right\} ; \lambda ; \zeta\right)$ implies that it satisfies the following recursive relation

$$
\begin{aligned}
F_{N}\left(\left\{u_{\alpha}\right\} ;\left\{\xi_{i}\right\} ; \lambda ; \zeta\right)= & \sum_{i=1}^{N} \frac{\sigma(\eta)}{\sigma\left(u_{N}-\xi_{i}+\eta\right) \sigma\left(u_{N}+\xi_{i}\right)} \prod_{l=1}^{N-1} \frac{\sigma\left(u_{l}-\xi_{i}\right) \sigma\left(u_{l}+\xi_{i}+\eta\right)}{\sigma\left(u_{N}-u_{l}\right) \sigma\left(u_{N}+u_{l}+\eta\right)} \\
& \times \prod_{j \neq i} \frac{\sigma\left(u_{N}-\xi_{j}\right) \sigma\left(u_{N}+\xi_{j}+\eta\right)}{\sigma\left(\xi_{j}-\xi_{i}\right) \sigma\left(\xi_{j}+\xi_{i}\right)} F_{N-1}\left(\left\{u_{\alpha}\right\}_{\alpha \neq N} ;\left\{\xi_{j}\right\}_{j \neq i} ; \lambda ; \zeta\right) .
\end{aligned}
$$

The determinant representation (5.9) and its recursive relation (5.12) of $F_{N}\left(\left\{u_{\alpha}\right\} ;\left\{\xi_{i}\right\} ; \lambda ; \zeta\right)$ and the recursive relation (5.11) of $B_{N}\left(\left\{u_{\alpha}\right\} ;\left\{\xi_{i}\right\} ; \lambda ; \zeta\right)$ imply that these two functions, as functions of $u_{N}$, have the same simple poles located at 3 :

$$
\xi_{i}-\eta,-\xi_{i} \quad \bmod \left(\Lambda_{\tau}\right), \quad i=1, \ldots, N
$$

Direct calculation shows that the residues of the two functions at each simple pole (5.13) are indeed the same

$$
\operatorname{Res}_{u_{N}=z}\left(F_{N}\left(\left\{u_{\alpha}\right\} ;\left\{\xi_{i}\right\} ; \lambda ; \zeta\right)\right)=\operatorname{Res}_{u_{N}=z}\left(B_{N}\left(\left\{u_{\alpha}\right\} ;\left\{\xi_{i}\right\} ; \lambda ; \zeta\right)\right), z=\xi_{i}-\eta,-\xi_{i}
$$

Let us introduce the function $f(u)$ :

$$
f\left(u_{N}\right)=F_{N}\left(\left\{u_{\alpha}\right\} ;\left\{\xi_{i}\right\} ; \lambda ; \zeta\right)-B_{N}\left(\left\{u_{\alpha}\right\} ;\left\{\xi_{i}\right\} ; \lambda ; \zeta\right) .
$$

The quasi-periodic properties of the $\sigma$-function,

$$
\sigma(u+1)=-\sigma(u), \quad \sigma(u+\tau)=-e^{-2 \sqrt{-1} \pi\left(u+\frac{\tau}{2}\right)} \sigma(u),
$$

\footnotetext{
${ }^{3}$ The determinant expression (5.9) guarantees that the apparent poles in (5.12), which are located at $u_{l},-u_{l}-\eta \bmod \left(\Lambda_{\tau}\right)$ for $l=1, \ldots, N-1$, do not really be poles.
} 
allow us to derive the following quasi-periodic properties of the function $f(u)$

$$
f(u+1)=f(u), \quad f(u+\tau)=e^{-2 \sqrt{-1} \pi(-2 u-\eta-\tau)} f(u) .
$$

This indicates [43] that the number of zero points minus that of poles of the function $f(u)$ inside the fundamental domain generated by 1 and $\tau$ should be -2 . However (5.13) and (5.14) imply that the function $f(u)$ is actually analytic inside the fundamental domain. Therefore, we can conclude that the function $f(u)$ must be zero function, i.e. $f(u)=0$. This means that (5.10) holds for $I=N$.

Hence we have completed the proof of (5.6).

Finally from the expression (5.3), we obtain the determinant representation of the partition function $Z_{N}\left(\left\{u_{\alpha}\right\} ;\left\{\xi_{i}\right\} ; \lambda ; \zeta\right)(2.13)$ of the eight-vertex model with a non-diagonal reflection end and the DW boundary condition

$$
\begin{aligned}
Z_{N}\left(\left\{u_{\alpha}\right\} ;\left\{\xi_{i}\right\} ; \lambda ; \zeta\right)= & \prod_{k=1}^{M} \frac{\sigma\left(\lambda_{12}+2 k \eta\right) \sigma\left(\lambda_{12}-2 k \eta+\eta\right)}{\sigma\left(\lambda_{12}+k \eta\right) \sigma\left(\lambda_{12}-k \eta+\eta\right)} \prod_{l=1}^{N} \prod_{i=1}^{N} \frac{\sigma\left(u_{i}+\xi_{l}\right)}{\sigma\left(u_{i}+\xi_{l}+\eta\right)} \\
& \times \frac{\prod_{\alpha=1}^{N} \prod_{i=1}^{N} \sigma\left(u_{\alpha}-\xi_{i}\right) \sigma\left(u_{\alpha}+\xi_{i}+\eta\right) \operatorname{det} \mathcal{N}\left(\left\{u_{\alpha}\right\} ;\left\{\xi_{i}\right\}\right)}{\prod_{\alpha>\beta} \sigma\left(u_{\alpha}-u_{\beta}\right) \sigma\left(u_{\alpha}+u_{\beta}+\eta\right) \prod_{k<l} \sigma\left(\xi_{k}-\xi_{l}\right) \sigma\left(\xi_{k}+\xi_{l}\right)},
\end{aligned}
$$

where the $N \times N$ matrix $\mathcal{N}\left(\left\{u_{\alpha}\right\} ;\left\{\xi_{i}\right\}\right)$ is given by (5.7).

\section{Conclusions}

We have studied the partition function $Z_{N}\left(\left\{u_{\alpha}\right\} ;\left\{\xi_{i}\right\} ; \lambda ; \zeta\right)$ of the eight-vertex model with a non-diagonal reflection end defined by the K-matrix (2.9) and the DW boundary condition. The DW boundary condition is specified by four boundary states (2.38)-(2.41). With the help of the F-basis provided by the Drinfeld twist for the eight-vertex SOS model, we obtain the complete symmetric expressions (5.3)-(5.4) of the partition function. Such an explicit expression allows us to derive the recursive relation (5.5). Solving the recursive relation, we find that the partition function $Z_{N}\left(\left\{u_{\alpha}\right\} ;\left\{\xi_{i}\right\} ; \lambda ; \zeta\right)$ can be represented as a single determinant (5.18), contrary to the eight-vertex model without reflection end [13, 14, 15]. Such a single determinant representation will be crucial for constructing the explicit expressions of the Bethe vectors and further investigation of scalar productions of the open XYZ chain with non-diagonal boundary terms [52]. 


\section{Acknowledgements}

The financial supports from the National Natural Science Foundation of China (Grant Nos. 11075126 and 11031005), Australian Research Council and the NWU Graduate Crossdiscipline Fund (08YJC24) are gratefully acknowledged.

\section{References}

[1] V. E. Korepin, Commun. Math. Phys. 86 (1982), 391.

[2] A. G. Izergin, Sov. Phys. Dokl. 32 (1987), 878.

[3] A. G. Izergin, D.A. Coker and V.E. Korepin, J. Phys. A25 (1992), 4315.

[4] F. H. L. Essler, H. Frahm, A. G. Izergin and V. E. Korepin, Commun. Math. Phys. 174 (1995), 191.

[5] V. E. Korepin, N. M. Bogoliubov and A. G. Izergin, Quantum Inverse Scattering Method and Correlation Functions, Cambridge University Press, 1993.

[6] N. Kitanine, J. M. Maillet and V. Terras, Nucl. Phys. B 554 (1999), 647.

[7] P.M. Bleher and V.V. Fokin, Exact solution of the six-vertex model with domain wall boundary conditions: Disordered phase, math-ph/0510033.

[8] K. Sogo, J. Phys. Soc. Jpn. 62 (1993) 1887.

[9] G. Kuperburg, Int. Math. Res. Not. 3 (1996) 139; Ann. of Math. 156 (2002), 835.

[10] A. Caradoc, O. Foda and N. Kitanine, J. Stat. Mech (2006), P03012.

[11] O. Foda, M. Wheeler and M. Zuparic, J. Stat. Mech. (2007), P10016; J. Stat. Mech. (2008), P02001.

[12] S.-Y. Zhao and Y.-Z. Zhang, J. Math. Phys. 48 (2007), 023504.

[13] S. Pakuliak, V. Rubtsov and A. Silantyev, J. Phys. A 41 (2008), 295204.

[14] W.-L. Yang and Y.-Z. Zhang, J. Math. Phys. 50 (2009), 083518 [arXiv:0903.3089]. 
[15] H. Rosengren, Adv. in Appl. Math. 43 (2009), 137.

[16] K. Hao, X. Chen, K.-J. Shi and W.-L Yang, Chin. Phys. B 20 (2011), 010303.

[17] O. Tsuchiya, J. Math. Phys. 39 (1998), 5946.

[18] E. K. Sklyanin, J. Phys. A 21 (1988), 2375.

[19] Y.-S. Wang, Nucl. Phys. B 622 (2002), 633.

[20] N. Kitanine, K. K. Kozlowski, J. M. Maillet, G. Niccoli, N. A. Slavnov and V. Terras, J. Stat. Mech. (2007), P10009.

[21] V. G. Drinfeld, Sov. Math. Dokl. 28 (1983), 667.

[22] J. M. Maillet and J. Sanchez de Santos, Drinfeld twists and algebraic Bethe ansatz, Amer. Math. Soc. Transl. 201 (2000), 137.

[23] R.I. Nepomechie, J. Stat. Phys. 111 (2003), 1363; J. Phys. A 37 (2004), 433.

[24] R. I. Nepomechie and F. Ravanini, J. Phys. A 36 (2003), 11391; Addendum, J. Phys. A 37 (2004), 1945.

[25] J. Cao, H.-Q. Lin, K.-J. Shi and Y. Wang, Nucl. Phys. B 663 (2003), 487.

[26] W.-L. Yang, Y.-Z. Zhang and M. Gould, Nucl. Phys. B 698 (2004), 312.

[27] J. de Gier and P. Pyatov, J. Stat. Mech. (2004), P03002; A. Nichols, V. Rittenberg and J. de Gier, J. Stat. Mech. (2005), P05003; J. de Gier, A. Nichols, P. Pyatov and V. Rittenberg, Nucl. Phys. B 729 (2005), 387.

[28] W.-L. Yang and R. Sasaki, Nucl. Phys. B 679 (2004), 495; J. Math. Phys. 45 (2004), 4301; W.-L. Yang, R. Sasaki and Y.-Z. Zhang, JHEP 09 (2004), 046.

[29] W. Galleas and M. J. Martins, Phys. Lett. A 335 (2005), 167; C.S. Melo, G. A. P. Ribeiro and M. J. Martins, Nucl. Phys. B 711 (2005), 565.

[30] J. de Gier and F. H. L. Essler, Phys. Rev. Lett. 95 (2005), 240601; J. Stat. Mech. (2006), P 12011.

[31] Z. Bajnok, J. Stat. Mech. (2006), P06010. 
[32] W.-L. Yang and Y.-Z. Zhang,JHEP 12 (2004), 019; JHEP 01 (2005), 021; W. -L. Yang, Y.-Z. Zhang and R. Sasaki, Nucl. Phys. 729 (2005), 594.

[33] A. Doikou and P. P. Martin, J. Stat. Mech. (2006), P06004; A. Dikou, J. Stat. Mech. (2006), P09010.

[34] R. Murgan, R. I. Nepomechie and C. Shi, J. Stat. Mech. (2006) P08006; R. Murgan, JHEP 04 (2009), 076.

[35] P. Baseilhac and K. Koizumi, J. Stat. Mech. (2007), P09006.

[36] W.-L. Yang, R. I. Nepomechie and Y.-Z. Zhang, Phys. Lett. B 633 (2006), 664; W.-L. Yang, and Y.-Z. Zhang, Nucl. Phys. B 744 (2006), 312; L. Frappat, R. I. Nepomechie and E. Ragoucy, J. Stat. Mech. (2007), P09008.

[37] W. Galleas, Nucl. Phys. B 790 (2008), 524.

[38] W.-L. Yang and Y.-Z. Zhang, JHEP 04 (2007), 044; Nucl. Phys. B 789 (2008), 591.

[39] L. Amico, H. Frahm, A. Osterloh and T. Wirth, Nucl. Phys. B 839 (2010), 604.

[40] N. Crampe, E. Ragoucy and D. Simon, e-print: arXiv: 1009.4119.

[41] G. Filali and N. Kitanine, J. Stat. Mech. 06 (2010), L06001; e-print: arXiv: 1011.0660.

[42] W.-L. Yang, X. Chen, J. Feng, K. Hao, B.-Y. Hou, K.-J. Shi and Y.-Z. Zhang, Nucl. Phys. B 844 (2011), 289.

[43] R. J. Baxter, Exactly solved models in statistical mechanics, Academic Press, New York, 1982.

[44] T. Inami and H. Konno, J. Phys. A 27 (1994), L913.

[45] B. Y. Hou, K. J. Shi, H. Fan and Z.-X. Yang, Commun. Theor. Phys. 23 (1995), 163.

[46] E. T. Whittaker and G. N. Watson, A course of modern analysis: 4th edn., Cambridge University Press, 2002.

[47] I. V. Cherednik, Theor. Math. Fiz 61 (1984), 35. 
[48] H. Fan, B.-Y. Hou, G.-L. Li and K.-J. Shi, Phys. Lett. A 250 (1998), 79.

[49] G. Felder and A. Varchenko, Nucl. Phys. B 480 (1996), 485.

[50] B. Y. Hou, R. Sasaki and W.-L. Yang, Nucl. Phys. B 663 (2003), 467; J. Math. Phys. 45 (2004), 559 .

[51] W.-L. Yang and Y.-Z. Zhang, Nucl. Phys. B 831 (2010), 408.

[52] W.-L. Yang, X. Chen, J. Feng, K. Hao, K.-J. Shi, Z.-Y. Yang and Y.-Z. Zhang, arXiv:1101.2935.

[53] T. -D. Albert, H. Boos, R. Flume, R. H. Poghossian and K. Rulig, Lett. Math. Phys. 53 (2000), 201.

[54] W.-L. Yang, X. Chen, J. Feng, K. Hao, B.-Y. Hou, K.-J. Shi and Y. -Z. Zhang, JHEP 01 (2011), 006. 